\title{
A Top-down Multi-scale Modeling for Actuation Response of Polymeric
}

\section{Artificial Muscles}

\author{
Qianxi Yang and Guoqiang Li* \\ Department of Mechanical \& Industrial Engineering \\ Louisiana State University \\ Baton Rouge, LA 70803, USA
}

\begin{abstract}
*Author to whom all correspondence should be addressed.
Tel.:001-225-578-5302; Fax: 001-225-578-5924; E-mail: Iguoqi1@lsu.edu
\end{abstract}

\begin{abstract}
A class of innovative artificial muscles made of high-strength polymeric fibers such as fishing lines or sewing threads have been discovered recently. These muscles are fabricated by a simple "twist insertion" procedure, which have attracted increasing attention due to their low cost and readily availability, giant tensile stroke, record energy density, and easy controllability. In the present paper, we established a multi-scale modeling framework for the thermomechanical actuation responses by a top-down strategy, spanning from macro-scale helical spring analysis down to molecular level chain interaction study. Comparison between modeling results and experimental results exhibited excellent agreement. The effect of the micro-, meso- and macroscale parameters on the actuation responses of the artificial muscle was further discussed through a parametric study per the validated model. This work helps understand the physical origin behind the remarkable tensile actuation behavior of the twisted-then-coiled polymeric artificial muscles and also provides inspirations for optimal design of advanced artificial muscles made by twist-insertion procedure.
\end{abstract}

Keywords: Artificial muscle; Multi-scale modeling; Actuation; Fiber; Top-down analysis. 


\section{Introduction}

Artificial muscles are a class of biologically inspired materials or devices that can reversibly contract, expand, or rotate by external stimuli, such as voltage (Ohm et al., 2010; Pelrine et al., 2002; Zhang et al., 1998), pressure (Chou and Hannaford, 1996; Daerden and Lefeber, 2002), current (Baughman, 1996; Lima et al., 2012; Shahinpoor et al., 1998), or temperature (Leng et al., 2011; Wayman, 1980). Recently, artificial muscles have become a popular topic in both academic and industrial areas: innovative actuation devices based on various mechanisms have been invented and advanced materials with performances surpassing those of natural muscles in many aspects have been developed.

The widely used dielectric elastomer actuator (DEA) is constructed based on the simple mechanism, that is, electrostatic attraction between conductive layers (Pelrine et al., 2002). Albeit a simple design principle with wide operating temperatures and large actuation strains, the high driven voltages $(\sim 1 \mathrm{kV})$ can be a concern, particularly in biomedical and toy applications. Actuators based on ferroelectric materials are realized by the application of an electric field, which results in permanently aligned and polarized domains until the Curie point is reached (Zhang et al., 1998). The high driven voltage, substantial hysteresis and difficulty in synthesis have become its key limitations. Liquid crystal elastomers (LCEs) exhibit good response times, and moderate to large strains under significantly lower voltage than that in ferroelectrics and dielectric elastomers (Ohm et al., 2010). However, since investigation of LCEs is still at a very early stage, lack of material information, low stiffness and high cost have become their main limitations. Actuators based on current, or movement of ions, such as conducting polymers (Baughman, 1996), ionic polymer metal composites (Shahinpoor et al., 1998), and hybrid carbon nanotube yarns (Lima et al., 2012) have demonstrated outstanding mechanical properties, but have limited reversibility and cycle rate. Other actuators, for instance, pneumatic artificial muscle (Chou and Hannaford, 1996), electro-thermally driven shape memory alloy (Wayman, 1980), and shape memory polymer (Leng et al., 2011), etc., all suffer from low scalability, high cost and limited cycle-ability. A comprehensive review of the emerging artificial muscle actuators can be found in the work by Madden et al. (2004) and Mirvakili et al. (2013). In summary, artificial muscles are of practical interest, but few types have been commercially exploited due to the various limitations discussed above. Therefore, an inexpensive, fast, non- 
hysteretic and scalable actuator, which can deliver high force with minimal weight and long cycle life, is highly desired to fulfil the requirement for various fields.

Recently, in a surprising breakthrough in the world of material science, Haines et al. (2014) have created new artificial muscles that can offer a hundredfold increase in power over natural muscles of equivalent lengths. They have demonstrated that the low cost fishing lines or sewing threads can actively contract and expand after being twisted then coiled into helical spring. The highest tensile stroke achieved was $34 \%$ for a temperature variation of $\sim 220^{\circ} \mathrm{C}$, compared with $4 \%$ strain for the untwisted fiber. Its giant tensile stroke, record energy density, quick response, and easy tuning procedure are some notable advantages of this novel class of artificial muscles. Subsequently, a series of experimental characterizations on the twisted fishing line fibers and coiled artificial muscles have been conducted by a number of researchers (Aziz et al., 2015; Cherubini et al., 2015; Moretti et al., 2015), aiming at providing information and useful data for future engineering applications. A custom experimental test-bench and procedure have been developed and employed by Moretti et al. (2015) to systematically run isothermal and isometric tensile tests on nylon-made coiled actuators (Cherubini et al., 2015). The results revealed some important issues related to the response of these actuators, such as hysteresis, repeatability, predictability and stored elastic energy. Aziz et al. (2015) standardized the methods for characterizing both the torsional stroke and torque generated, and various testing modes were studied, which replicated possible application conditions: free stroke in a one-end tethered fiber, torsional actuation against an externally applied torque, blocked rotation to determine the generated toque, and torsional actuation against a return spring fiber. Because the muscles can be indefinitely long, they could find uses in both miniaturized and macroscopic actuators. The robustness, commercial availability, and low cost of the polymeric muscles further confirm the significance of this innovative discovery and has opened new horizons toward the development of effective devices, for instance, morphing airplanes and vehicles (McEvoy and Correll, 2015), self-healing composite (Zhang and Li, 2015; Zhang and Li, 2016), robotics (Yip and Niemeyer, 2015), etc.

It is worth mentioning that the concept of twisting then coiling a fiber for the purpose of actuation is not limited to the polymeric fiber. It has also been conducted previously on carbon nanotube yarns (Foroughi et al., 2011; Lima et al., 2012) and niobium nanowires (Mirvakili et al., 2013). However, they exhibited much lower performance than fishing lines did. In their work, 
the authors explained the torsional actuation behavior from purely kinematic point of view, where the rotation angle was expressed simply by geometrical parameters, i.e., yarn diameter, length, and volume.

Several studies have been conducted to explain this extraordinary thermomechanical behavior of twisted-then-coiled polymeric muscles, which facilitate future design. In recognition of the similarities between the maximum specific work during torsional actuation for a noncoiled twisted fiber and that during tensile contraction for a twisted-then-coiled spring, Haines et al. (2014) pointed out that the large-stroke tensile actuation of the coiled spring arises from the torsional actuation of the twisted polymer fiber. In addition, the authors used a rough analysis and proved that the thermally triggered torsional actuation for the twisted fiber was attributed to both the contraction in axial direction and expansion in transverse direction of the precursor fiber. In addition, the relation between the coil length and the fiber twists per unit length was established from purely kinematic point of view using Love equation (Love, 1944) and Van Der Heijdgen and Thompson theory (Van der Heijden and Thompson, 2000). Most recently, Sharafi and $\mathrm{Li}$ (2015) developed a physics-based constitutive framework, in which the actuation response of the twisted-then-coiled spring was considered to be determined by two types of astrained molecules, that is, helical chains and entropic chains. However, the involvement of a large number of molecular scale parameters in the constitutive framework, which are difficult to determine, makes the use of the molecular scale model of considerable challenge. Therefore, they also proposed a computationally efficient phenomenological thermomechanical constitutive model, which was largely dependent on curve fittings. Because of this, the phenomenological model is unable to pin down the underlying mechanisms controlling the muscle actuation behavior.

In summary, there is still a lack of theoretical models to elucidate the physical origin behind the remarkable tensile actuation behavior and, therefore, the correlation between the actuation performance and the intrinsic material parameters remains unclear. In the present paper, we will analyze the actuation response from macro-scale helical spring top-down to the molecular chain interaction. We will build a multi-scale modeling framework, in order to reproduce and predict the actuation behavior and evaluate the impact of various factors on the response of polymeric artificial muscles. This paper is arranged as follows. Section 1 introduces the background and motivations for this study. In Section 2, a general top-down analysis is given. 
This is followed by the development of a multi-scale modeling framework in Section 3. The model validation and parametric study are conducted in Section 4. In Section 5, the important results and findings are summarized.

\section{Top-down Analysis}

This twisted-then-coiled artificial muscle contains hierarchical structures and it is schematically illustrated in Fig. 1.

The coiled muscles (Fig. 1(a)) are able to reversibly delivery over 20\% contraction upon heating and lift heavy load (Haines et al., 2014), which is an abnormal behavior that cannot be explained by the classical spring mechanism. More specifically, Eq. (1) is the classical relation of the axial applied load $F$ and the displacement $\delta$ of the spring, in terms of the properties of the fiber (shear modulus $G_{f}$ and diameter $d_{f}$ ) and geometries of the coil (mean diameter $\overline{D_{c}}$ and number of active coils $n$ ) (Shigley et al., 1989):

$$
F=\frac{G_{f} d_{f}^{4}}{8{\overline{D_{c}}}^{3} n} \delta
$$

Based on Eq. (1), since the shear modulus drops as temperature increases, the coil will elongate, instead of contracting upon heating. The essential reason behind this contradiction is the twisted configuration of the fiber, as shown in Fig. 1(b). This highly twisted structure exhibits large torsional actuation when heated and hence generates large twisting moment when tethered at both ends. Intuitively, in a coil made of this twisted fiber, the released torque can screw the coil segments, compete with the external applied load and eventually result in the contraction of the coil.

As suggested by Haines et al. (2014), the torsional actuation is mostly attributed to both the thermal contraction in the axial direction and thermal expansion in the radial direction of the precursor fiber, which are both at least an order of magnitude smaller than that of the coiled spring (See the coefficients of thermal expansion presented in Fig. 1 on the left). In order to reproduce the torsional behavior of the twisted fiber, the thermomechanical properties of the twisted fiber are necessary. For an anisotropic polymer fiber, studies have shown that twisting has a significant impact on the thermomechanical properties of fibers or yarns (Ma et al., 2014; Militký and Kovačič, 2011; Sze et al., 1976) and a number of models have been developed to elucidate this dependence (Pipes and Hubert, 2002, 2003; Suhai, 1983; Zhao et al., 2014). A commonly used approach is decomposing the twisted fiber into concentric hollow cylinders, and 
then each hollow cylinder is hypothetically cut and opened up into a layered structure as shown in Fig. 1(c). Considering the fact that most of the polymeric fibers used to make artificial muscles are semi-crystalline consisting of aligned micro-fibrils, each layer of the twisted fiber contains collimated micro-fibrils, whose orientation is determined by the position of the layer from the cylinder center axis, namely, the outermost layer has the most oriented fibrils and the innermost layer has no orientation. This configuration is very much analogous to the fiberreinforced composite. As such, the thermomechanical properties of the off-axis laminate (Fig. 1(c)) and the assembly of the lamina with different angles (Fig. 1(b)), i.e. the twisted fiber, can be obtained once the properties of the on-axis lamina (Fig. 1(d)) are given based on composite material theory, together with three dimensional helical anisotropic cylinder analysis.

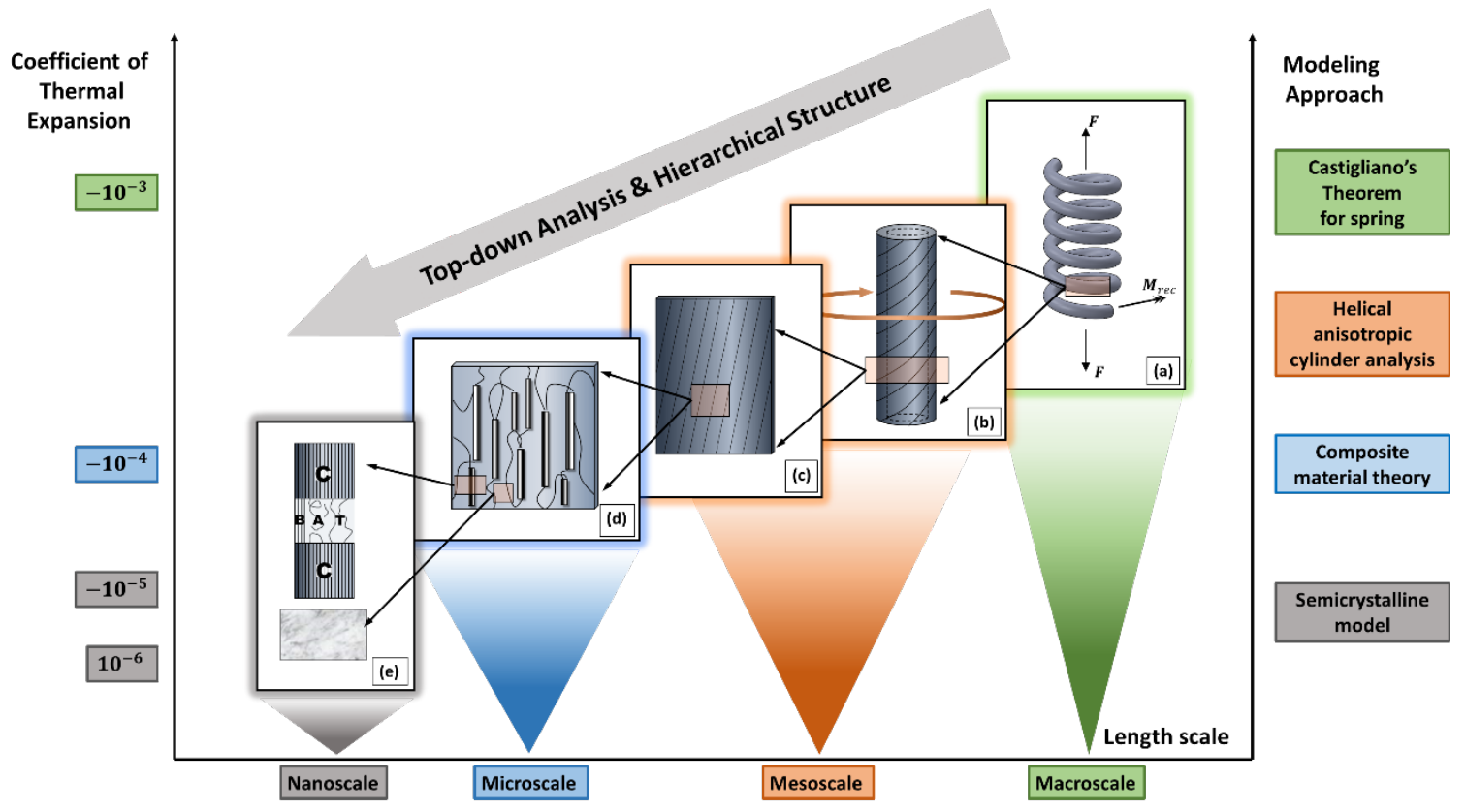

Fig. 1. Multi-scale modeling framework: (a) Twisted then coiled spring. (b) A section of the twisted fiber. (c) Off-axis laminate, hypothetically cut and separated from the twisted fiber. (d) On-axis lamina, consisting of discontinuous aligned micro-fibrils embedded into an amorphous matrix. (e) Top: crystal blocks connected in series with intercrystalline materials. Bottom: amorphous matrix made of rubbery and glassy phases.

The analyses of the on-axis lamina properties are case dependent. Most commonly used precursor fibers are semi-crystalline fibers, such as Polyethylene and Nylon 6. In our current study, we focus on artificial muscle made of Polyethylene (PE) copolymer monofilament under the brand name of ZEBCO OMNIFLEX30LBA, due to its convenient availability. A closer observation by previous researchers (Barham and Arridge, 1977; Choy and Leung, 1985; Grubb 
and Prasad, 1992; Kip et al., 1991) reveals that in a highly oriented Polyethylene fiber, the crystalline would form needlelike, discontinuous micro-fibrils with the long axis in the fibrils direction, and are embedded into an amorphous matrix phase (See Fig. 1(d)). The amorphous matrix (Bottom inset of Fig. 1(e)), of which the coefficient of thermal expansion (CTE) is positive (See Fig. 1 on the left for the CTE of the amorphous matrix), can always be regarded as a mixture of glassy phase and rubbery phase with temperature dependent volume fractions. As for the micro-fibrils (Top inset of Fig. 1(e)), they have been described as crystal blocks with negative CTE of the order of $-10^{-5} K^{-1}$ (denoted as part $\mathbf{C}$ in the upper inset of Fig. 1(e)), periodically connected in series with inter-crystalline materials, which may consist of a parallel combination of inter-crystalline bridges (denoted as part $\mathbf{B}$ in the upper inset of Fig. 1(e)), amorphous chains (denoted as part $\mathbf{A}$ in the upper inset of Fig. 1(e)), and tie molecules (denoted as part $\mathbf{T}$ in the upper inset of Fig. 1(e)) (Baughman, 1973; Choy et al., 1981).

In summary, we divided the multi-scale modeling within the continuum mechanics framework into four levels: (1) Macro-scale model: In this length scale, we focus on the relation between the applied load and the displacement of the spring. The polymeric fiber, of which the coiled spring is made, has a diameter of $\sim 1 \mathrm{~mm}$, and possesses no discernable microstructural features, i.e., it is completely homogenized. (2) Meso-scale model: At this length scale, the twisted fiber is treated as a stacked laminate, while the material within each lamina is assumed to be homogenized and transversely isotropic. This assumption requires an appropriate thickness for the lamina. Firstly, the thickness should be small enough, so that the twisting orientation angle $\left(\theta_{f}\right)$ distribution within each layer is not too broad and using an averaged orientation angle to represent the corresponding ply is acceptable. Meanwhile, the thickness should also be large enough, in order to include sufficient amount of micro-fibrils within its cross-section. The crystalline micro-fibrils from the micro-scale perspective are orthotropic materials. Therefore, only when there are enough micro-fibrils in the cross-section, can we apply the homogenized and transversely isotropic assumptions to each lamina. For the polyethylene fiber we are studying, previous study has reported that the intrinsic length scale for the thickness of the micro-fibrils are of the order of $15 \mathrm{~nm}$ and $\sim 200 \mathrm{~nm}$ for the length (Grubb and Prasad, 1992). Therefore, we believe that a lamina thickness of the order of $\sim 1 \mu \mathrm{m}$ is an appropriate choice for our meso-scale modeling. (3) Micro-scale model: In this case, the micro-structure of each lamina is taken into account. Classical continuum elasticity based on homogenization may fail at this scale due to the 
size effect (Maranganti and Sharma, 2007). Maranganti and Sharma (2007) demonstrated that the characteristic length scale at which the size effect come into play for polymer systems ranges from $30 \mathrm{~nm}$ to $150 \mathrm{~nm}$, which is comparable to the intrinsic length scale reported for the crystalline micro-fibrils in the polyethylene fiber. Within the micromechanics framework, we thus treat each lamina as a two-phase composite consisting of discontinuous crystalline microfibrils embedded into the amorphous matrix. A factor will be introduced later to account for the imperfect interfacial interaction between the two phases. (4) Nano-scale model: The fine structures of each component (crystalline micro-fibrils and amorphous matrix) in micro-scale model are further considered. The material at this length scale may contain a variety of topological defects and chemical impurities which may significantly alter its properties. Polyethylene, as one of the commonly used polymer, has been extensively studied. Therefore, we will directly utilize the results from existing literatures and provide inputs for the micro-scale model.

Notice that, starting from the crystal blocks, which are bridged by the twisted semicrystalline fiber and finally to the coiled spring, the thermal contractions are magnified progressively based on different mechanisms. In the next section, we will build up a multi-scale modeling framework for each step of the foregoing analyses.

\section{Multi-scale Modeling Framework}

\subsection{Macro-scale modeling for the twisted-then-coiled spring}

Previous study (Haines et al., 2014) has suggested that the remarkable tensile actuation behavior is driven by the giant torsional actuation generated by the twisted fiber during heating. We here will quantify this mechanism based on a consistent application of Castigliano's second theorem (CST) (Dym, 2009). The coordinate systems, which include global Cartesian $\left(\left\{\boldsymbol{X}_{\boldsymbol{c}}, \boldsymbol{Y}_{\boldsymbol{c}}, \boldsymbol{Z}_{\boldsymbol{c}}\right\}\right)$, global cylindrical $\left(\left\{\boldsymbol{r}_{\boldsymbol{c}}, \boldsymbol{\vartheta}_{\boldsymbol{c}}, \boldsymbol{z}_{\boldsymbol{c}}\right\}\right)$, and local cylindrical coordinate $\left(\left\{\boldsymbol{r}_{\boldsymbol{f}}, \boldsymbol{\vartheta}_{\boldsymbol{f}}, \boldsymbol{z}_{\boldsymbol{f}}\right\}\right)$, used to analyze the helical coil, are shown in Fig. 2(b). Throughout the working cycle, a constant load $\boldsymbol{F}=F \boldsymbol{Z}_{\boldsymbol{c}}$ is applied to the coil. In addition, since both ends of the twisted fiber are tethered, the untwisting tendency of the fiber is fully constrained during the working cycle, ending up with a recovered torque in the axial direction along the twisted fiber, $\boldsymbol{M}_{r e c}=M_{r e c} \boldsymbol{z}_{f}$. Notice that the direction of the recovered torque is determined by chirality of the twisted-coil: A homo-chiral 
coil, that is, the chirality of the fiber twist matches that of the coil, leads to recovered torque in $z_{f}$ direction, while a hetero-chiral coil results in recovered torque in $-z_{f}$ direction.

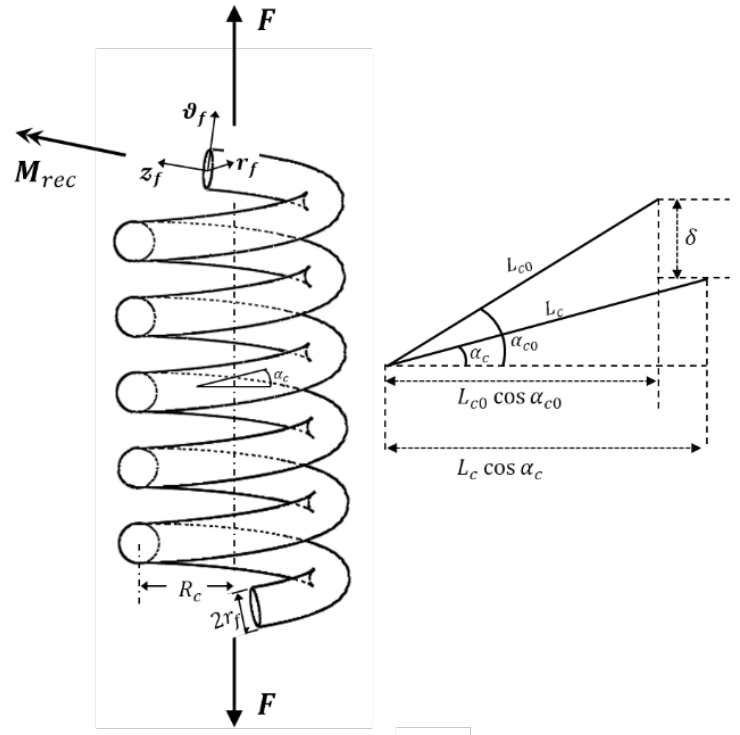

(a)

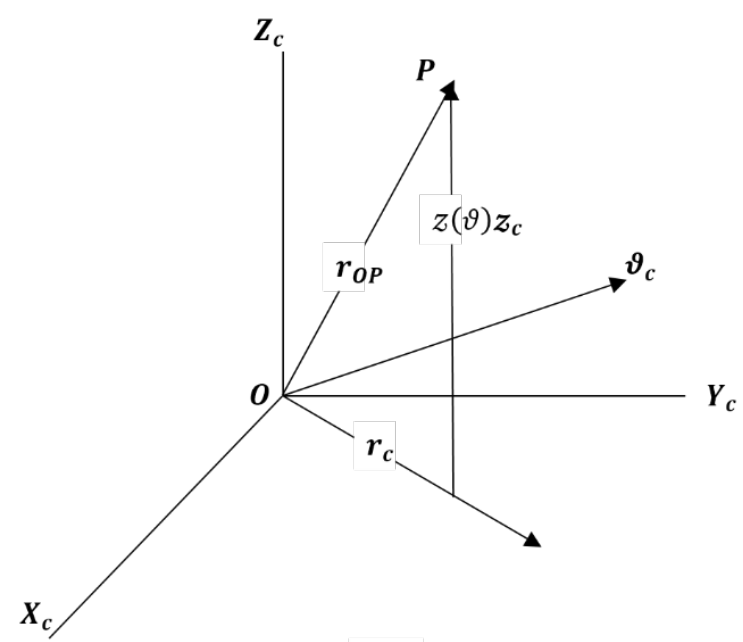

(b)

Fig. 2. (a) Left: Coiled spring loaded with applied force $\boldsymbol{F}$ and recovered torque $\boldsymbol{M}_{\text {rec }}$. Right: Kinematic relationship of the coiled spring. (b) The coordinate systems for the coiled spring that are used in the model. (Definitions of the notations are given in the text.)

For a given applied force $\boldsymbol{F}=F \boldsymbol{Z}_{\boldsymbol{c}}=F \boldsymbol{z}_{\boldsymbol{c}}$, by using the relationship between the local and global coordinates:

$$
\begin{gathered}
\boldsymbol{r}_{f}=-\boldsymbol{r}_{\boldsymbol{c}} \\
\boldsymbol{\vartheta}_{\boldsymbol{f}}=-\sin \alpha_{c} \boldsymbol{\vartheta}_{c}+\cos \alpha_{c} \boldsymbol{z}_{c} \\
\boldsymbol{z}_{\boldsymbol{f}}=\cos \alpha_{c} \boldsymbol{\vartheta}_{\boldsymbol{c}}+\sin \alpha_{c} \boldsymbol{z}_{\boldsymbol{c}}
\end{gathered}
$$

where $\boldsymbol{r}_{\boldsymbol{f}}$ is the unit vector in the coil's cross section pointing inward, $\boldsymbol{z}_{\boldsymbol{f}}$ is the unit vector along the coil's tangent direction, and $\boldsymbol{\vartheta}_{\boldsymbol{f}}$ is the unit vector in the plane of the fiber's cross section as dictated by the vector product $\boldsymbol{\vartheta}_{\boldsymbol{f}}=\boldsymbol{z}_{\boldsymbol{f}} \times \boldsymbol{r}_{\boldsymbol{f}}$, and $\alpha_{c}$ is the pitch angle of the coil. The applied force on the slightly inclined, positively directed face at point $\boldsymbol{P}$ can be expressed in terms of the local coordinate:

$$
\boldsymbol{F}_{a p p}=\boldsymbol{F}=F \sin \alpha_{c} \boldsymbol{z}_{\boldsymbol{f}}+F \cos \alpha_{c} \boldsymbol{\vartheta}_{\boldsymbol{f}}
$$


The free body diagram shows that the applied force also results in torsional and bending moment at point $\boldsymbol{P}$ :

$$
\boldsymbol{M}_{a p p}^{F}=\boldsymbol{r}(P O) \times(-\boldsymbol{F})=-F R \boldsymbol{\vartheta}_{c}=-F R \cos \alpha_{c} \boldsymbol{z}_{\boldsymbol{f}}+F R \sin \alpha_{c} \boldsymbol{\vartheta}_{\boldsymbol{f}}
$$

The recovery torque, $\boldsymbol{M}_{\text {rec }}=M_{r e c} \boldsymbol{z}_{f}$ is considered to be applied on top of $\boldsymbol{M}_{a p p}^{F}$, thus, the total applied moment at point $\boldsymbol{P}$ is:

$$
\boldsymbol{M}_{a p p}=\boldsymbol{M}_{a p p}^{F}+\boldsymbol{M}_{r e c}=\left(-F R \cos \alpha_{c}+M_{r e c}\right) \boldsymbol{z}_{f}+F R \sin \alpha_{c} \boldsymbol{\vartheta}_{\boldsymbol{f}}
$$

For convenience, we re-write Eqs. (3) and (5) as:

$$
\begin{gathered}
\boldsymbol{F}_{a p p}=F_{z} \boldsymbol{z}_{\boldsymbol{f}}+F_{\vartheta} \boldsymbol{\vartheta}_{\boldsymbol{f}} \\
\boldsymbol{M}_{a p p}=M_{z} \boldsymbol{z}_{\boldsymbol{f}}+M_{\vartheta} \boldsymbol{\vartheta}_{\boldsymbol{f}}
\end{gathered}
$$

with the normal force $F_{z}=F \sin \alpha_{c}$, shear force $F_{\vartheta}=F \cos \alpha_{c}$, torsional moment $M_{z}=$ $-F R \cos \alpha_{c}+M_{\text {rec }}$ and bending moment $M_{\vartheta}=F R \sin \alpha_{c}$. These components are constant along the coil. By assuming that, at each step of temperature increment, the deformations along the fiber caused by the external load are infinitesimal, the complementary energy can be expressed as (Clive and Shames, 1973):

$$
\begin{aligned}
& U^{*}=\int_{0}^{L_{c}}\left(\frac{M_{z}^{2}}{2 \bar{G} J}+\frac{M_{\vartheta}^{2}}{2 \bar{E} I}+\frac{F_{z}^{2}}{2 \bar{E} A}+\frac{F_{\vartheta}^{2}}{2 \bar{G} A}\right) d l \\
& =L_{c}\left(\frac{M_{z}^{2}}{2 \bar{G} J}+\frac{M_{\vartheta}^{2}}{2 \bar{E} I}+\frac{F_{z}^{2}}{2 \bar{E} A}+\frac{F_{\vartheta}^{2}}{2 \bar{G} A}\right) \\
& =\frac{1}{2} f_{11} F^{2}-2 f_{12} F M_{r e c}+\frac{1}{2} f_{22} M_{r e c}^{2}
\end{aligned}
$$

where $\bar{E}$ and $\bar{G}$ are the effective moduli of the twisted fiber and

$$
\begin{gathered}
f_{11}=\frac{1}{2} L_{c}\left(\frac{1}{\bar{G} J} R^{2} \cos ^{2} \alpha_{c}+\frac{1}{\bar{E} I} R^{2} \sin ^{2} \alpha_{c}+\frac{1}{\bar{G} A} \cos ^{2} \alpha_{c}+\frac{1}{\bar{E} A} \sin ^{2} \alpha_{c}\right) \\
f_{12}=L_{c} \frac{R \cos \alpha_{c}}{2 \bar{G} J} \\
f_{22}=L_{c} \frac{1}{\bar{G} J}
\end{gathered}
$$


Taking the cross-section area of the fiber $A=\frac{\pi d^{2}}{4}$, the second moment $I=\frac{\pi d^{4}}{64}$, the polar moment of that area $J=\frac{\pi d^{4}}{32}$ and the radius of the coil $R=\frac{D}{2}=\frac{L_{c} \cos \alpha_{c}}{n \pi}$, Eq. 8 becomes:

$$
\begin{gathered}
f_{11}=\frac{8 n}{\pi^{3} d^{4}}\left(\frac{L_{c}}{n}\right)^{3} \frac{\cos ^{4} \alpha_{c}}{\bar{G}}+\frac{8 n}{\pi d^{2}}\left(\frac{L_{c}}{n}\right) \frac{\cos ^{2} \alpha_{c}}{2 \bar{G}} \\
+\frac{8 n}{\pi^{3} d^{4}}\left(\frac{L_{c}}{n}\right)^{3} \frac{2 \sin ^{2} \alpha_{c} \cos ^{2} \alpha_{c}}{\bar{E}}+\frac{8 n}{\pi d^{2}}\left(\frac{L_{c}}{n}\right) \frac{\sin ^{2} \alpha_{c}}{2 \bar{E}} \\
f_{12}=\frac{8 n}{\pi^{2} d^{4}}\left(\frac{L_{c}}{n}\right)^{2} \frac{\cos ^{2} \alpha_{c}}{\bar{G}} \\
f_{22}=\frac{32}{\pi d^{4}} \frac{L_{c}}{\bar{G}}
\end{gathered}
$$

Then, the displacement $\delta$ of a helical spring is directly found by applying CST (Dym, 1997):

$$
\delta=f_{11} F-f_{12} M_{r e c}
$$

The displacement can also be expressed as a function of the pitch angle $\alpha_{c}$ using the kinematic relationship:

$$
\delta=L_{c} \sin \alpha_{c}-L_{c 0} \sin \alpha_{c 0}
$$

where $L_{c 0}$ and $\alpha_{c 0}$ are the initial length of the fiber and the initial pitch angle of the coil (after loading), respectively. Using Eqs. (10) and (11), the pitch angle $\alpha_{c}(T)$, hence the displacement $\delta(T)$ can be expressed in terms of $d, L_{c}, \bar{G}, \bar{E}, M_{\text {rec }}$, etc., on which temperature dependences will be determined using the meso-scale modeling described in the following section.

\subsection{Meso-scale modeling for the thermomechanical behavior of the twisted fiber}

\subsubsection{Concentric helically anisotropic laminate}

As stated in Section 2, the twisted fiber is decomposed into concentric hollow cylinders, and then hypothetically cut and separated into a lamina containing collimated micro-fibrils (See Fig. 3).

To avoid confusion and complexity, we first clarify the symbols and notations. Referring to Fig. 3, the principal material axes are labeled as $\mathbf{1}$ and $\mathbf{2}$, where $\mathbf{1}$-axis is parallel to the micro- 
fibrils and 2 -axis is normal to them; $\boldsymbol{\vartheta}_{\boldsymbol{f}}$ and $\boldsymbol{z}_{\boldsymbol{f}}$ are principal terms of the off-axis behavior. According to the definition in classical lamination theory and the handedness of the coordinate, the off-axis angle $\theta_{f}$ is depicted in Fig. 3.

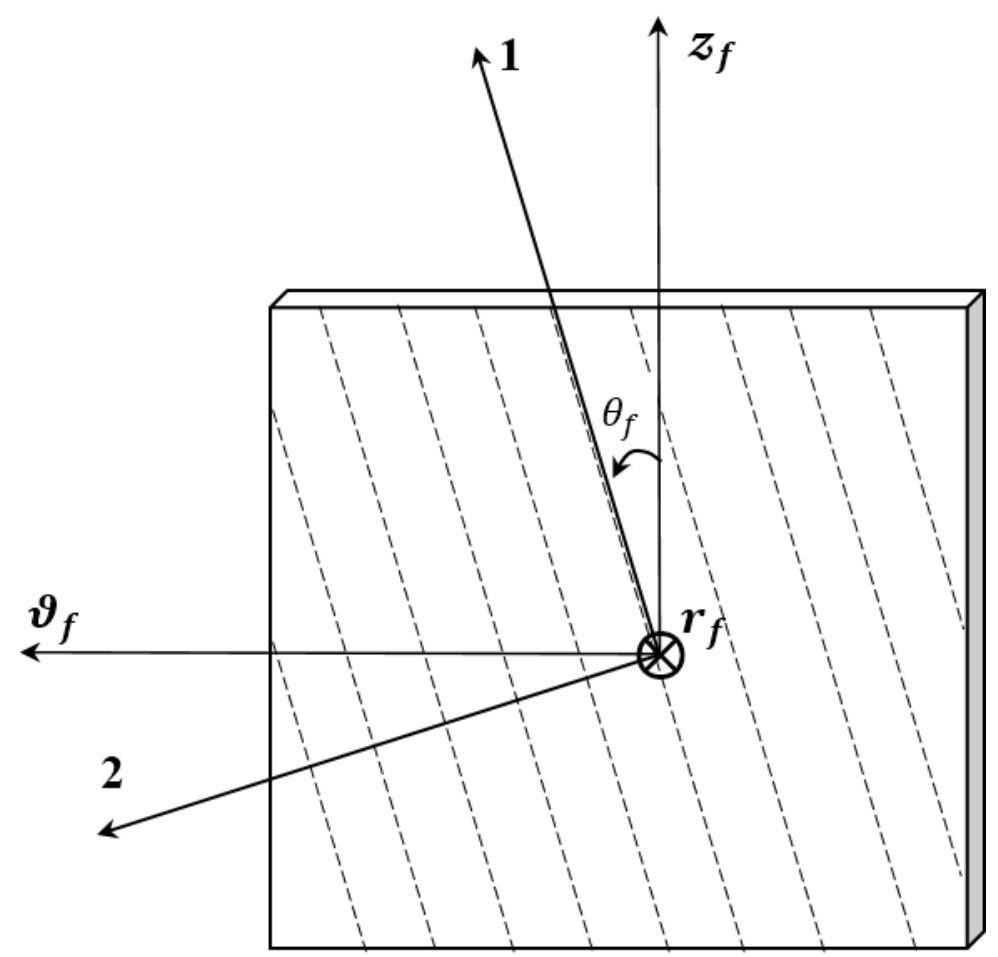

Fig. 3. Opened-up structure of the micro-fibrils reinforced composite lamina.

Once we have obtained the on-axis transverse symmetric thermomechanical properties, i.e. the stiffness matrix $\mathcal{C}$ and the coefficients of thermal expansion $\alpha^{\prime} s$, which will be determined in the next section, we are able to evaluate the off-axis laminate using Eqs. (12) and (13). 


$$
\left\{\begin{array}{c}
\bar{C}_{11}=C_{11} m^{4}+2\left(C_{12}+C_{16}\right) m^{2} n^{2}+C_{22} n^{4} \\
\bar{C}_{12}=\left(C_{11}+C_{22}-4 C_{66}\right) m^{2} n^{2}+C_{12}\left(m^{4}+n^{4}\right) \\
\bar{C}_{13}=C_{13} m^{2}+C_{23} n^{2} \\
\bar{C}_{16}=-C_{22} m n^{3}+C_{11} m^{3} n-\left(C_{12}+2 C_{66}\right) m n\left(m^{2}-n^{2}\right) \\
\bar{C}_{22}=C_{11} n^{4}+2\left(C_{12}+2 C_{66}\right) m^{2} n^{2}+C_{22} m^{4} \\
\bar{C}_{23}=C_{13} n^{2}+C_{23} m^{2} \\
\bar{C}_{26}=-C_{22} m^{3} n+C_{11} m n^{3}+\left(C_{12}+2 C_{66}\right) m n\left(m^{2}-n^{2}\right) \\
\bar{C}_{33}=C_{33} \\
\bar{C}_{36}=\left(C_{13}-C_{23}\right) m n \\
C_{66}=\left(C_{11}+C_{22}-2 C_{12}\right) m^{2} n^{2}+C_{66}\left(m^{2}-n^{2}\right)^{2}
\end{array}\right.
$$

and

$$
\left\{\begin{array}{c}
\alpha_{z}=\alpha_{1} m^{2}+\alpha_{2} n^{2} \\
\alpha_{\theta}=\alpha_{1} n^{2}+\alpha_{2} m^{2} \\
\alpha_{r}=\alpha_{3}=\alpha_{2} \\
\alpha_{z \theta}=2\left(\alpha_{1}-\alpha_{2}\right) m n
\end{array}\right.
$$

where $m=\cos \theta_{f}$, and $n=\sin \theta_{f}$. Notice that the appearance of a coupling term $\alpha_{z \theta}$ in Eq. (13), indicates that a reversible thermal shear strain will be generated during heating or cooling. In other words, the torsional behavior of the twisted fiber can be explained intuitively from the composite material point of view. The procedures to obtain displacements $\{u, v, w\}$ and stress components $\left\{\sigma_{z}, \sigma_{\theta}, \sigma_{r}, \sigma_{z \theta}\right\}$ for each layer are detailed in Appendix A. In the next sub-section, we will assemble all the layers and determine the effective properties for the twisted fiber.

\subsubsection{Multi-layer analysis}

Suppose the cylinder consists of $N$ layers of hollow cylinders. In order to clarify the position of each layer, we hereafter put a superscript ' $k$ ' to each component, for example, $\sigma_{z}^{k}, u^{k}$ and $\theta_{f}^{k}$. The inner most layer has an inner radius of $r^{0}$ and outer most layer has an outer radius of $r^{N}$ (See Fig. 4(b)). $r^{0}$ is taken to be a number very small, for example $10^{-10} \mathrm{~mm}$, in order to simulate a solid cylinder and meanwhile to avoid singularity. Then, the outer radius of the $k^{\text {th }}$ layer becomes $r^{k}=r^{0}+k \frac{r_{f}-r^{0}}{N}$, where $r_{f}=r^{N}$, is the radius of the fiber. Also, the tangent of the helix angle is assumed to vary linearly with radial position, reaching its maximum at the 
outer layer of the cylinder, that is, at the $k^{t h}$ layer $\theta_{f}^{k}(r)=\arctan \frac{r^{k}}{r_{f}} \tan \alpha_{f}$, where $\alpha_{f}$ is the helical angle at the surface of the fiber.

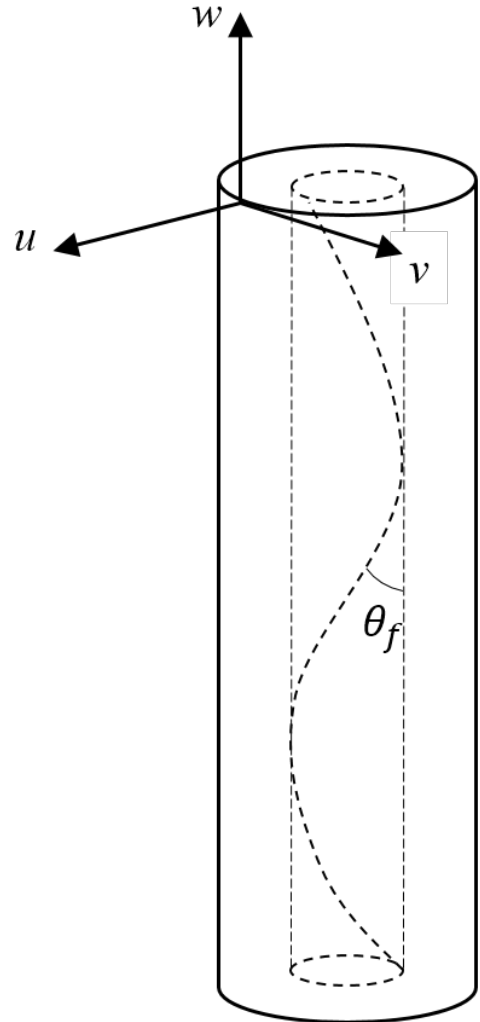

(a)
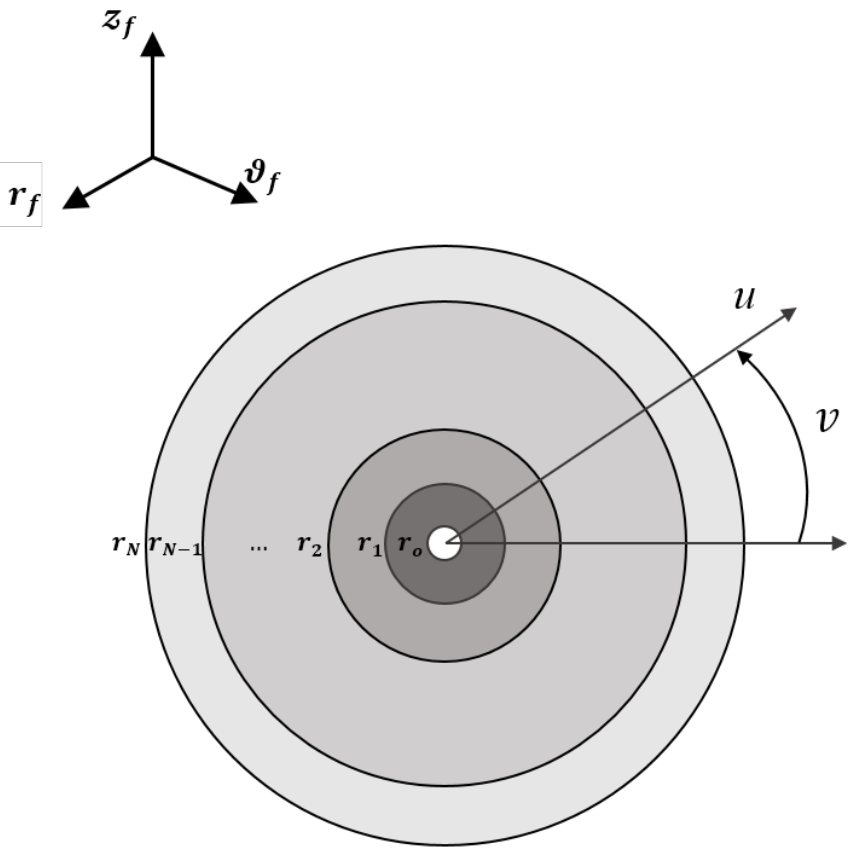

(b)

Fig. 4. Multi-layer analysis

The continuity and boundary conditions then require:

$$
\begin{gathered}
\left\{\begin{array}{c}
\sigma_{r}^{1}\left(r_{0}\right)=0 \\
\left\{\begin{array}{l}
\sigma_{r}^{1}\left(r_{1}\right)=\sigma_{r}^{2}\left(r_{1}\right) \\
u^{1}\left(r_{1}\right)=u^{2}\left(r_{1}\right)
\end{array}\right. \\
\left\{\begin{array}{c}
\sigma_{r}^{k-1}\left(r_{1}\right)=\sigma_{r}^{k}\left(r_{1}\right) \\
u^{k-1}\left(r_{1}\right)=u^{k}\left(r_{1}\right) \\
\ldots
\end{array}\right. \\
\sigma_{r}^{N}\left(r_{N}\right)=0
\end{array}\right.
\end{gathered}
$$


Using these $2 N$ boundary conditions, the $2 N$ undetermined coefficients $C_{1}^{k}$ and $C_{2}^{k}$ (See Appendix A) in each layer can be determined, and hence the stress and strain distributions on the cross section of the twisted fiber can be obtained.

The determination of the effective thermomechanical properties that is needed for the coil analysis, i.e. $\overline{E_{z}}, \overline{G_{z \theta}}, \overline{\alpha_{z}}, \overline{\alpha_{\theta}}$, and $\overline{\alpha_{z \theta}}$, is accomplished by using the method of relaxation that has been described in the work by Pipes and Hubert (2003). Generally speaking, at a certain condition $\left(v_{0}, w_{0}, \Delta T\right)$, the resultant torque $\tau$ and force $f$ can be expressed using the influence coefficients:

$$
\left[\begin{array}{ll}
A & B \\
C & D
\end{array}\right]\left[\begin{array}{l}
v_{0} \\
w_{0}
\end{array}\right]=\left[\begin{array}{l}
\tau \\
f
\end{array}\right]
$$

where,

$$
\tau=2 \int_{r^{0}}^{r_{f}} \sigma_{\theta z} \pi r^{2} d r \quad f=2 \int_{r^{0}}^{r_{f}} \sigma_{z} \pi r d r
$$

and

$$
\left\{\begin{array}{l}
A=\tau\left(v_{0}=1, w_{0}=0, \Delta T=0\right) \\
B=\tau\left(v_{0}=0, w_{0}=1, \Delta T=0\right) \\
C=f\left(v_{0}=1, w_{0}=0, \Delta T=0\right) \\
D=f\left(v_{0}=0, w_{0}=1, \Delta T=0\right)
\end{array}\right.
$$

The effective mechanical properties are then straightforward:

$$
\left\{\begin{array}{l}
\overline{E_{z}}=\frac{f\left(v_{0}=-\frac{B}{A} w_{0}, w_{0}, \Delta T=0\right)}{\pi r_{f}^{2} w_{0}} \\
\overline{G_{z \theta}}=\frac{2 \tau\left(v_{0}, w_{0}=-\frac{C}{D} v_{0}, \Delta T=0\right)}{\pi r_{f}^{4} v_{0}}
\end{array}\right.
$$

For the effective thermal expansion, we first set the strain components $v_{0}$ and $w_{0}$ to be zero, and the thermal torque $\tau_{T}$ and force $f_{T}$ are determined for a given temperature increment $\Delta T$. Notice that $\tau_{T}$ is the recovered torque increment in the temperature step $\Delta T$, the total recovered torque at a certain temperature is the accumulation of each temperature step:

$$
M_{\text {rec }}=\int_{T_{0}}^{T} \tau_{T} d T
$$


Next, the corresponding thermal strain components are determined using:

$$
\left[\begin{array}{ll}
A & B \\
C & D
\end{array}\right]\left[\begin{array}{l}
v_{0 T} \\
w_{0 T}
\end{array}\right]=\left[\begin{array}{l}
-\tau_{T} \\
-f_{T}
\end{array}\right]
$$

Finally,

$$
\left\{\begin{array}{l}
\overline{\alpha_{z}}=\frac{w_{0} T}{\Delta T} \\
\overline{\alpha_{\theta}}=\frac{u_{0}}{\Delta T} \\
\overline{\alpha_{z \theta}}=\frac{v_{0} R}{\Delta T}
\end{array}\right.
$$

where, $u_{0}=u\left(r_{f}\right)$.

\subsection{Nano- and Micro-scale modeling for the semi-crystalline polyethylene fiber}

The microscopic structures for the semi-crystalline polyethylene fiber have been described in Section 2 and are depicted in Fig. 5. Fig. 5(a) presents the phenomenological representation for the aforementioned repeated structure unit in a crystalline micro-fibrils: A crystal block $\mathbf{C}$ is connected in series with $\mathbf{S}$ (which is a collective representation of bridges (b) and amorphous molecules (a)) and tie molecules (T). In the following statement, the properties of the crystal block are defined as $X^{C}$, likewise, properties of crystalline bridges, amorphous molecules, component $\mathbf{S}$ and tie molecules are defined as $X^{b}, X^{a}, X^{S}$, and $X^{T}$, respectively. Regarding the micro-fibrils, and the amorphous phase, the properties are designated as $X^{f}$ and $X^{m}$, respectively. ( $X$ could be moduli, CTEs, and volume fractions, etc.) 


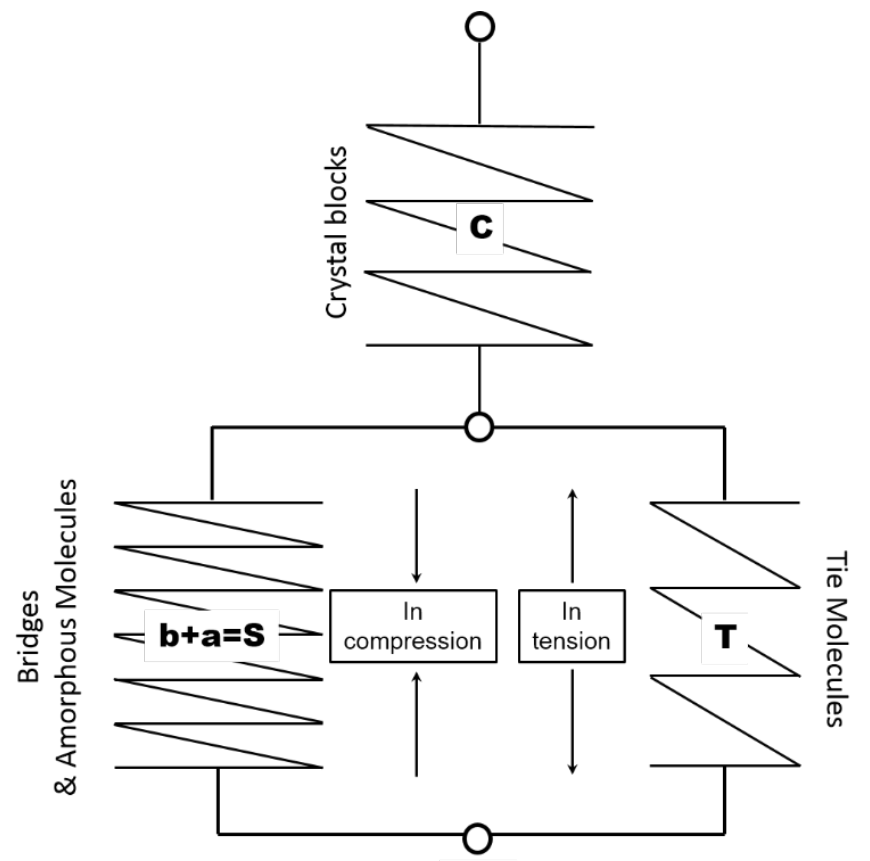

(a)

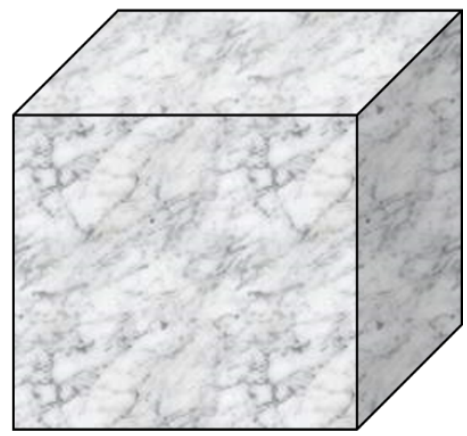

(b)

Fig. 5. Phenomenological representation for (a) crystalline micro-fibrils and (b) amorphous matrix.

For the block crystals, both theoretical calculations (He, 1986; Klei and Stewart, 1986; Odajima and Maeda, 1967; Pechhold and Blasenbrey, 1970; Suhai, 1983; Tashiro et al., 1978) and experimental measurements (Chan et al., 1978; Choy and Leung, 1985; Grubb and Prasad, 1992; Holliday and White, 1971; Kip et al., 1991; Rider and Watkinson, 1978) have been performed to evaluate their elastic moduli $C_{i j}^{c}$. We here assume that within the working cycle, these elastic moduli are temperature independent. This can be justified by the fact that the melting temperature of the fiber is $220^{\circ} \mathrm{C}$ (See Fig. 7), which is well above the highest working temperature $\left(80^{\circ} \mathrm{C}\right)$ for the muscle. To account for the weakening effect of the inter-crystalline materials on the mechanical performance of the micro-fibril in the axial direction, we simply introduce a scale factor $\lambda$ to the modulus in the axial direction and leave the transverse direction almost unchanged:

$$
\begin{gathered}
E_{1}^{f}=\frac{C_{11}^{c}}{\lambda} \\
E_{2}^{f}=\frac{C_{22}^{c}+C_{33}^{c}}{2} \\
G_{12}^{f}=C_{66}^{c}
\end{gathered}
$$


Although the inter-crystalline materials do not show special effect on the mechanical properties, they play decisive roles in amplifying the negative thermal expansion capability of the crystal blocks. Take polyethylene as an example, it is well known that the coefficient of thermal expansion (CTE) for the crystalline block and bridge is of the order of $-1 \times 10^{-5} \mathrm{~K}^{-1}$ (Davis et al., 1970; Kobayashi and Keller, 1970), and is positive for the amorphous domains (Choy et al., 1979). However, the whole material may exhibit a CTE of the order of $-1 \times$ $10^{-4} K^{-1}$ (in axial direction) after drawing (Baughman, 1973; Choy et al., 1981). Therefore, the large negative CTE of the polymer fiber does not origin from the intrinsic material properties of each components, it is essentially attributed to the "internal strain" between the crystalline bridges and the tie molecules. More specifically, the natural length of the bridge is longer than that of the tie molecule. When they are in parallel, the bridges will be compressed and the tie molecule will be tensioned (See Fig. 5(a)). In addition, the tie molecules obey entropic elasticity while the elasticity for the bridge is almost constant. With a suitable volume fraction ratio between the two components, the overall material can exhibit large amount of contraction upon heating. As a matter of fact, this phenomenon is found to be common in essence with the shape memory effect: they are both related to the residual internal strain or stored strain induced by drawing or programming process. In Choy et al. (1981), the authors have performed a quantitative treatment and proved this effect. Without going into further details, the CTE in the axial direction for the micro-fibril can be expressed as:

$$
\alpha_{\|}^{f}=x^{C} \alpha_{\|}^{C}+\left(1-x^{C}\right) \alpha_{\|}^{S}-\left(1-x^{C}\right) \frac{d \eta}{d T}
$$

where $\alpha_{\|}^{C}$ and $\alpha_{\|}^{S}$ are the CTEs of the crystal block $\mathbf{C}$ and component $\mathbf{S}$ (See Fig. 5(a)), respectively; $\eta=\frac{t E_{1}^{T}}{b E_{1}^{c}} \propto T$ and $t, b$ and $x^{C}$ are the volume fraction of the tie molecules, bridges and block crystals within the micro-fibrils.

Fig. 5(b) shows a well-accepted phenomenological model of an amorphous phase. The amorphous phase is always treated as a mixture of glassy phase and rubbery phase, on which volume fractions are temperature dependent. In other words, a glassy phase may evolve into rubbery phase upon heating. This phase transition does not take place in all glassy phases at once, however, starts from the domains with higher energy and evolves gradually until the transition is fully accomplished (Yang and Li, 2015). To address this behavior, we adopt the phase evolution 
law introduced by Guo et al. (2015) to describe the temperature dependence of the glassy phase volume fraction within the amorphous matrix:

$$
x_{g}^{m}(T)=\int_{T_{0}}^{T} \frac{1}{S \sqrt{2 \pi}} e^{-\frac{\left(T-T_{g}\right)^{2}}{2 S^{2}}} d T
$$

where $S$ is a constant and $T_{g}$ represents the glass transition temperature.

By assuming that the amorphous phase is isotropic and with uniform stress distribution throughout the material, the thermomechanical properties are given by:

$$
\begin{gathered}
G_{12}^{m}=\frac{1}{x_{g}^{m} \frac{1}{G_{g}^{m}}+\left(1-x_{g}^{m}\right) \frac{1}{G_{r}^{m}}} \\
E_{1}^{m}=E_{2}^{m}=2 G_{12}^{m}\left(1+v_{12}^{m}\right) \\
\alpha_{\|}^{m}=\alpha_{\perp}^{m}=x_{g}^{m} \alpha_{g}^{m}+\left(1-x_{g}^{m}\right) \alpha_{r}^{m}
\end{gathered}
$$

where $x_{g}^{m}$ is the volume fraction of the glassy domain within the amorphous phase.

Having obtained the thermomechanical properties for the crystalline micro-fibrils and the amorphous matrix, the on-axis thermomechanical properties that are needed for Eqs. (12) and (13) can be obtained by noticing the resemblance of the current configuration to the short fiber reinforced composite (See Fig. 1(d)). According to the shear lag theory first developed by Cox (1952), the effective axial Young's modulus $E_{1}$ of a composite containing a discontinuous reinforcing phase of length, $l^{f}$, radius, $r^{f}$, Young's modulus, $E_{1}^{f}$, volume fraction, $V^{f}$, and matrix phase of Young's modulus $E_{1}^{m}$, shear modulus $G_{12}^{m}$, is:

$$
\begin{gathered}
E_{1}=V^{f} K E_{1}^{f}\left(1-\left(\frac{\tanh (x)}{x}\right)\right)+\left(1-V^{f}\right) E_{1}^{m} \\
x=\frac{l^{f}}{r^{f}}\left(\frac{G_{12}^{m}}{E_{1}^{f} \ln \left(2 \pi / \sqrt{3} V^{f}\right)}\right)^{\frac{1}{2}}
\end{gathered}
$$

with the knowledge that the load transfer between the crystalline micro-fibrils and the amorphous matrix is less than perfect, a constant $K$ is included in the Cox analysis, which is a measurement of the reinforcing efficiency (Pipes and Hubert, 2002; Pipes and Hubert, 2003).

The transverse Young's Modulus $E_{2}$ and in-plane shear modulus $G_{12}$ can be obtained by the handy equations developed by Halpin and Tsai (Halpin, 1969; Halpin and Kardos, 1976):

$$
\frac{P}{P^{m}}=\frac{1+\xi \eta V^{f}}{1-\eta V^{f}}
$$




$$
\eta=\frac{\frac{P^{f}}{P^{m}}-1}{\frac{P^{f}}{P^{m}}+\xi}
$$

here, $P^{f}$ is the micro-fibrils property, $P^{m}$ is the matrix property and $\xi$ is a measurement of the reinforcement geometry, which depends on loading conditions. The values of $\xi$ for $E_{2}$ and $G_{12}$ are 0.5 and 1 , respectively.

As for the CTEs, by assuming the simple "rule of mixtures”, we have:

$$
\alpha=V^{f} a^{f}+\left(1-V^{f}\right) \alpha^{m}
$$

\section{Model Validation and Prediction}

\subsection{Parameter identification}

The parameters for the multi-scale modeling were determined through various thermomechanical testing, values in the existing literatures, and appropriate curve fittings.

\subsubsection{Polymeric artificial muscle preparation}

The fabrication of artificial muscles follows the same procedure as in Haines et al. (2014), which takes two consecutive steps: twist insertion and coiling. The detailed fabrication procedures can be found in the work by Zhang and Li (2015). After the fabrication, the geometries of the precursor fiber and twisted fiber were measured using a SEM imaging system (Quanta $^{\mathrm{TM}}$ 3D FEG field-emission electron microscope) and those of the coiled spring were measured using an optical high resolution CCD camera (Sony XCD-CR90) (See Fig. 6). The sample codes, the fabrication descriptions, and the geometrical parameters for different samples that will be used in the modeling are summarized in Table 1.

Table 1. Summary of samples

\begin{tabular}{lll}
\hline Sample code & Fabrication description & Geometrical parameters \\
\hline Sample 0 & $\begin{array}{l}\text { Precursor fiber, polyethylene } \\
\text { copolymer. }\end{array}$ & $d_{0}=0.58 \mathrm{~mm}$ \\
Sample 1T & $\begin{array}{l}\text { Twist the fiber under 13.36MPa load } \\
\text { until coil nucleation, then anneal the } \\
\text { twisted fiber at } 85^{\circ} \mathrm{C} \text { for } 90 \text { min. }\end{array}$ & $\begin{array}{l}d_{1}=0.78 \mathrm{~mm} \\
\alpha_{f}=35^{\circ}\end{array}$ \\
Sample 2TC & $\begin{array}{l}\text { Twist the fiber under } 13.36 \mathrm{MPa} \text { load } \\
\text { until coil nucleation, wrap the twisted } \\
\text { fiber around a mandrel, then anneal the } \\
\text { coiled muscle at } 85^{\circ} \mathrm{C} \text { for } 90 \text { min. }\end{array}$ & $\begin{array}{l}D_{c}=2.11 \mathrm{~mm} \\
\alpha_{c}=10^{\circ} \\
n=8\end{array}$ \\
\hline
\end{tabular}




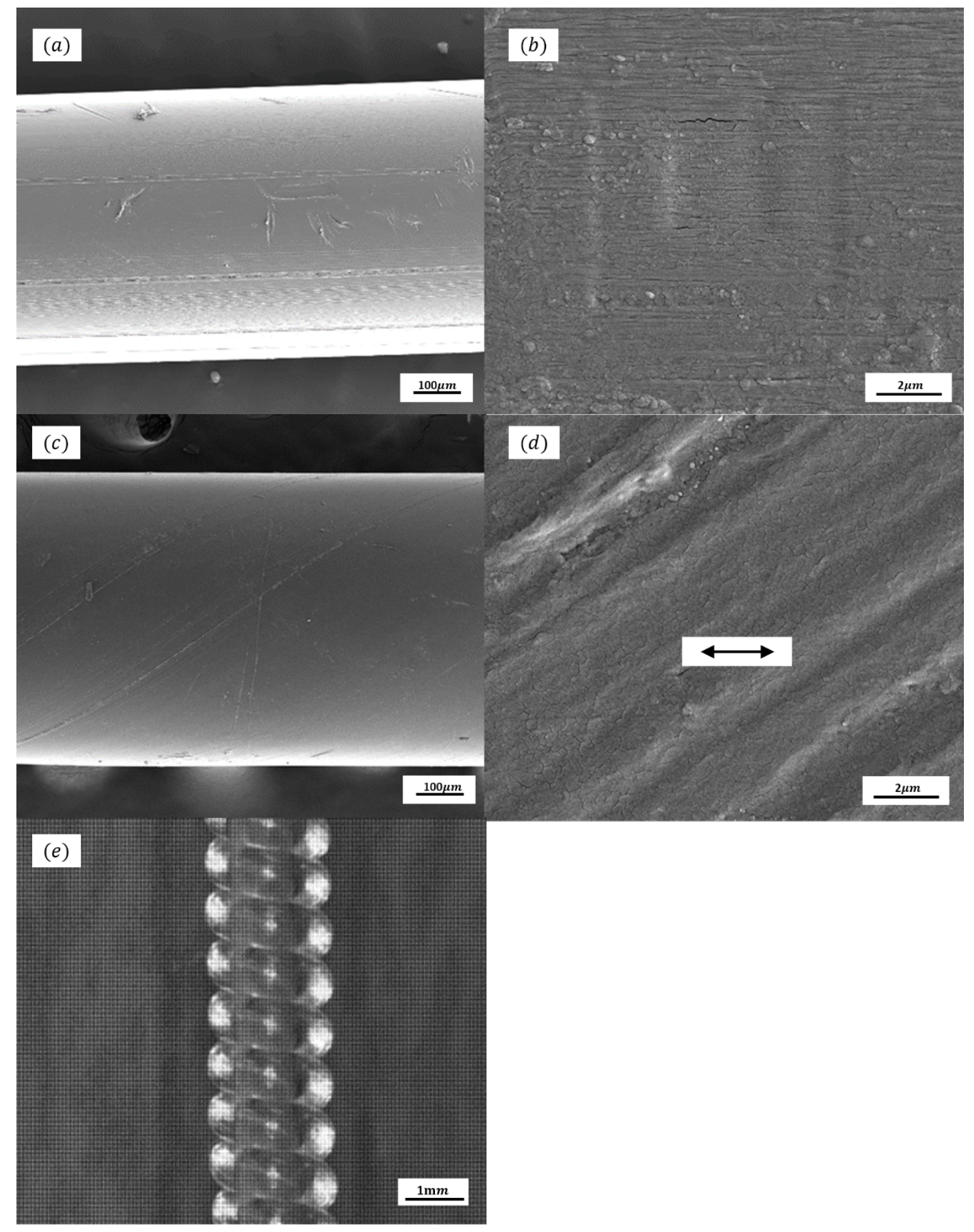

Fig. 6. (a) and (b) SEM images of the precursor fiber. (c) and (d) SEM images of the twisted fiber. (e) CCD image of the coiled spring. 


\subsubsection{Thermomechanical characterizations}

To investigate the morphologies of the fibers and provide experimental evidence for our model, the SEM observation were conducted on both Sample 0 and Sample 1T. As suggested by previous studies (Sze et al., 1976), the high strength and stiffness of polymer fibers are mostly attributed to their highly oriented crystalline morphologies. This is confirmed by the image in Fig. 6(b). Although we are not able to accurately measure the volume fraction $V^{f}$ and aspect ratio $l_{f} / r_{f}$ of the micro-fibrils in our samples (in Eq. (26)), values of 0.75 and 15, respectively, are believed to be appropriate as suggested by previous studies (Barham and Arridge, 1977; Grubb and Prasad, 1992; Kip et al., 1991). In addition, it is clear from Fig. 6(d) that after the twisting and heat-setting procedures, the micro-fibrils form off-axis configuration as described in our model.

Differential scanning calorimetry (PerkinElmer DSC 4000, USA) was used to investigate the thermal properties of the fishing lines. Samples were cooled from $30^{\circ} \mathrm{C}$ to $-70^{\circ} \mathrm{C}$ at $10^{\circ} \mathrm{C} / \mathrm{min}$ and heated from $-70^{\circ} \mathrm{C}$ to $240^{\circ} \mathrm{C}$, also at $10^{\circ} \mathrm{C} / \mathrm{min}$. Then, this thermal cycle was repeated. The first cycle DSC results for both Sample 0 and Sample $1 \mathrm{~T}$ are presented in Fig. 7, together with the second cycle DSC results for both samples as a comparison. The results again indicate that the morphology of the fiber consists of two phases: (I) Soft amorphous phase, represented by the broad glass transition region starts from around $20^{\circ} \mathrm{C}$ and ends around $60^{\circ} \mathrm{C}$. (II) Hard crystalline phase, represented by the melting peak at $220^{\circ} \mathrm{C}$. No significant change can be observed between Sample 0 and Sample 1T, indicates that the twisting procedure does not interrupt the morphology of the fiber very much and a working cycle between the room temperature $\left(25^{\circ} \mathrm{C}\right)$ and $80^{\circ} \mathrm{C}$ is appropriate for this fishing line. For the second cycle DSC curve, multiple and less perfect melting peaks can be observed, an indication of the less oriented crystalline structure in the raw material compared with the highly oriented fishing line in the first cycle (the first cycle has erased the working history of the fiber and thus both samples show the same DSC curve in the second cycle). The glass transition temperature used in our model is chosen to be $T_{g}=30^{\circ} \mathrm{C}$. 


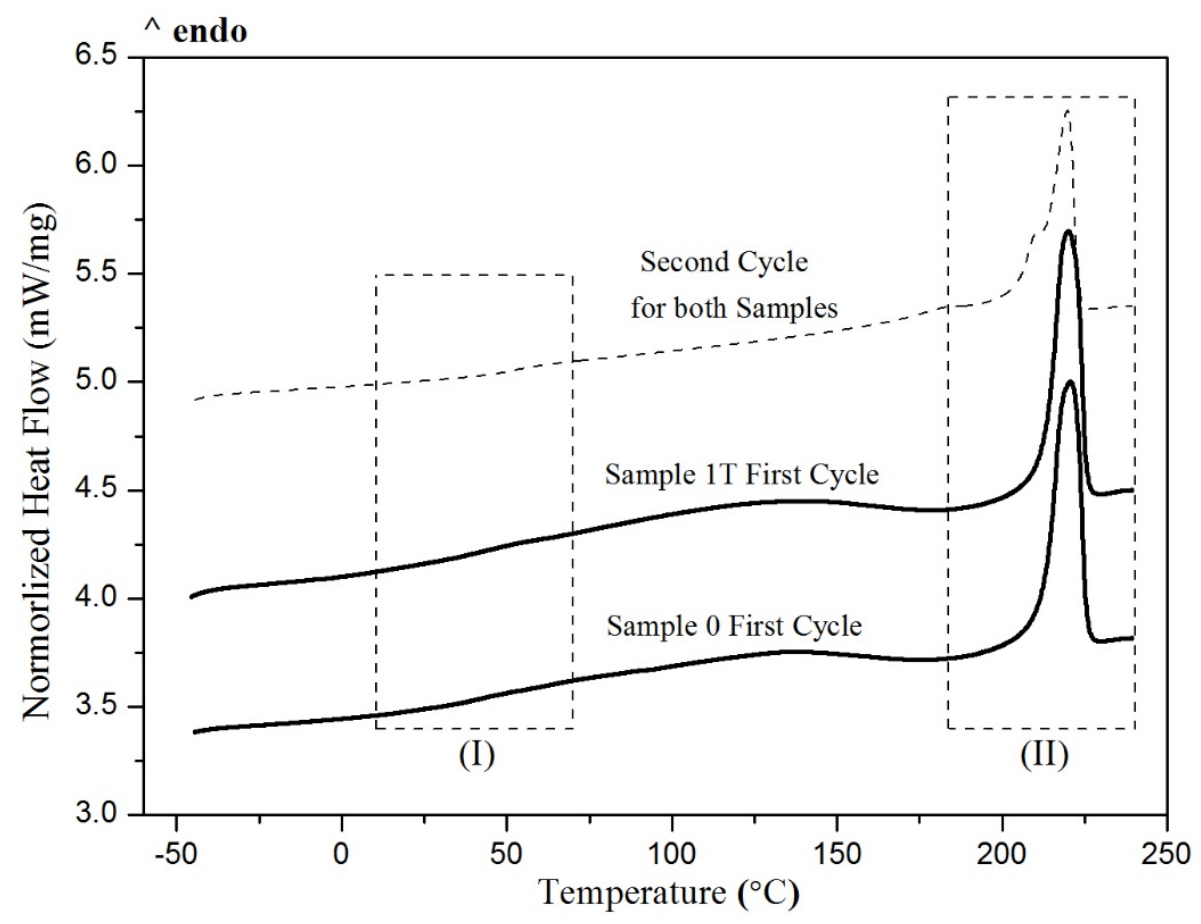

Fig. 7. DSC results for Sample 0 and Sample 1T.

A TA Instrument Dynamic Mechanical Analyzer (Q800) was used to perform a strain controlled dynamic temperature scan at a frequency of $1 \mathrm{~Hz}$. A $0.5 \%$ oscillating strain was applied to Sample 0 and Sample $1 \mathrm{~T}$ and the temperature was scanned from $25^{\circ} \mathrm{C}$ to $80^{\circ} \mathrm{C}$ at $1^{\circ} \mathrm{C} / \mathrm{min}$. The temperature dependence of the storage modulus of Sample 0 is presented in Fig. 8 and this result is used to identify the parameters in Eqs. (25)-(27). The elastic moduli for the crystal blocks are obtained from the stiffness constants of orthorhombic polyethylene crystal calculated by Tashiro et al. (1978) and those of amorphous matrix are approximated using the experimental results by Boyd (1979) and Xiong et al. (2015); the rest of the parameters in microscale modeling, i.e. $\lambda, K$ and $S$ (in Eqs. 25-27) can be varied and determined by fitting into the experimental results (See Fig. 8 upper lines). In addition to that, as evidenced by Sample 1T in Fig. 8, the axial modulus has been decreased after twisting due to disorientation. 


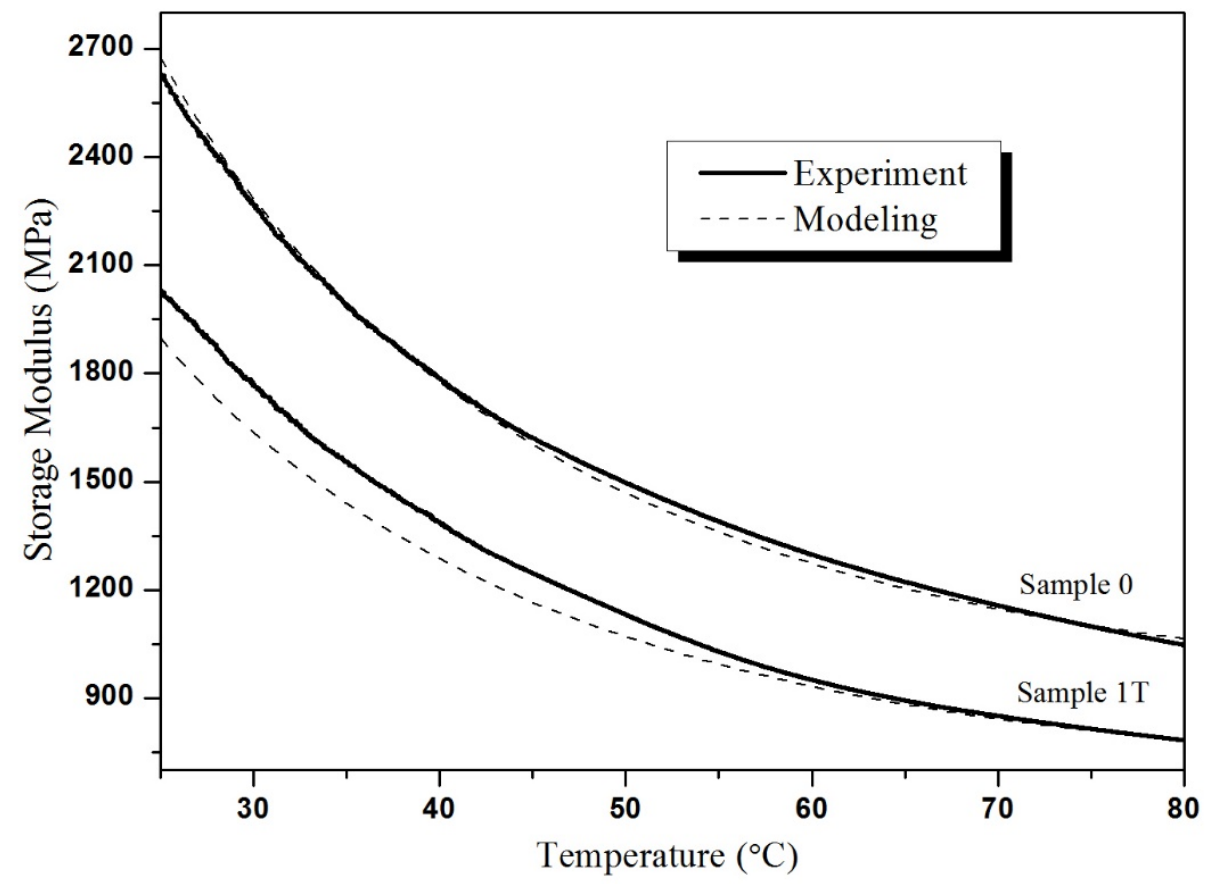

Fig. 8. Temperature dependences of axial moduli for both Sample 0 and Sample 1T (Solid lines: DMA experiment results. Dash lines: modeling results).

A temperature scan test under constant static force was also conducted on Sample 0 in order to measure the coefficient of axial thermal expansion $\left(\alpha_{1}\right)$. In our study, $\alpha_{1}$ is assumed to be linear temperature dependent, and accordingly, the displacement is a quadratic function in terms of temperature (See Fig. 9(a)). The coefficient of transverse thermal expansion $\left(\alpha_{2}\right)$ is not easy to measure, so we extract the experimental results for a similar material from Choy et al. (1981). Likewise, $\alpha_{2}$ is simulated by assuming a linear temperature dependence (Fig. 9(b)). 

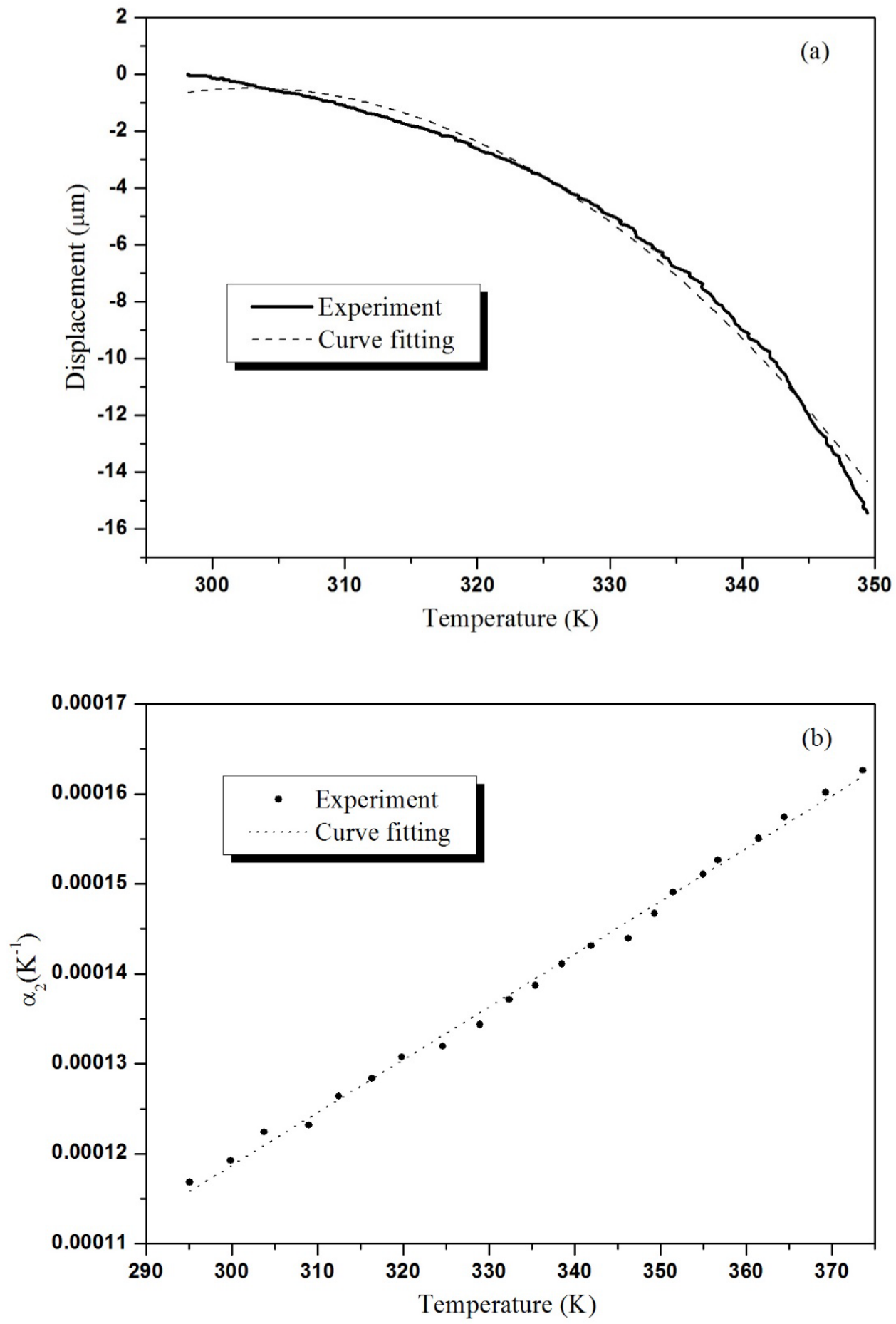

Fig. 9. Experimental and curve fitting results for the thermal properties of the precursor fiber in axial (a) and (b) transverse directions (The experimental data were extracted from Choy et al. (1981)). 


\subsubsection{Summary of parameters}

Up to now, we have completed the multi-scale modeling and determined the input parameters for the nano- and micro-, meso- and macro- scale modeling using experimental measurements, literature research and curve fitting procedures. In Table 2, all the parameters, together with their description and identification method, have been specified. The multi-scale model was then coded and implemented into the MATLAB program to reproduce the thermomechanical behavior of the twisted fiber and the coiled spring.

Table 2. Parameters used for the present multi-scale model.

\begin{tabular}{|c|c|c|c|}
\hline Parameters & Descriptions & Values & Identification Method \\
\hline \multicolumn{4}{|c|}{ Parameters for nano- and micro-scale modeling } \\
\hline$C_{11}^{C}(M P a)$ & $\begin{array}{l}\text { Stiffness of the crystal block in } \\
\text { fiber direction (Eq. 22(a)) }\end{array}$ & 300,000 & Tashiro et al., 1978 \\
\hline$\lambda$ & $\begin{array}{l}\text { Scale factor to account for the } \\
\text { weakening effect of the inter- } \\
\text { crystalline materials to the whole } \\
\text { micro-fibrils (Eq. 22(a)) }\end{array}$ & 3.25 & $\begin{array}{l}\text { Fit into DMA } \\
\text { experimental results } \\
\text { (Fig. } 8 \text { upper lines) }\end{array}$ \\
\hline$E_{2}^{f}(M P a)$ & $\begin{array}{l}\text { Young's modulus of the micro- } \\
\text { fibrils in transvers direction (Eq. } \\
\text { 22(b)) }\end{array}$ & 8,000 & Tashiro et al., 1978 \\
\hline$l_{f} / r_{f}$ & $\begin{array}{l}\text { Aspect ratio of the micro-fibrils } \\
\text { (Eq. 26(a)) }\end{array}$ & 15 & Grubb and Prasad, 1992 \\
\hline$K$ & $\begin{array}{l}\text { Measurement of the reinforcing } \\
\text { efficiency (Eq. 26(a)) }\end{array}$ & 0.85 & $\begin{array}{l}\text { Fit into DMA } \\
\text { experimental results } \\
\text { (Fig. } 8 \text { upper lines) }\end{array}$ \\
\hline$V^{f}$ & $\begin{array}{l}\text { Volume fraction of the crystalline } \\
\text { micro-fibrils (Eq. } 26 \text { and 27) }\end{array}$ & 0.75 & $\begin{array}{l}\text { Barham and Arridge, } \\
1977\end{array}$ \\
\hline$S$ & $\begin{array}{l}\text { Constants in the phase evolution } \\
\text { law (Eq. 24) }\end{array}$ & 40 & $\begin{array}{l}\text { Fit into DMA } \\
\text { experimental results } \\
\text { (Fig. 8. upper lines) }\end{array}$ \\
\hline$T_{g}\left({ }^{\circ} \mathrm{C}\right)$ & $\begin{array}{l}\text { Glass transition temperature (Eq. } \\
\text { 24) }\end{array}$ & 30 & DSC results (Fig. 7) \\
\hline$G_{g}^{m}(M P a)$ & $\begin{array}{l}\text { Shear modulus of the amorphous } \\
\text { matrix in the glassy state (Eq. } \\
25(a))\end{array}$ & 90 & Boyd, 1979 \\
\hline$v_{12}^{m}$ & $\begin{array}{l}\text { Poisson's ratio of the amorphous } \\
\text { matrix (Eq. 25(b)) }\end{array}$ & 0.4 & Boyd, 1979 \\
\hline
\end{tabular}




\begin{tabular}{|c|c|c|c|}
\hline \multicolumn{4}{|c|}{ Parameters for meso-scale modeling } \\
\hline$\alpha_{1}\left(K^{-1}\right)$ & Axial direction CTE & $\begin{array}{l}-1.35 \times 10^{-6} \mathrm{~T}+ \\
4.20 \times 10^{-4}\end{array}$ & $\begin{array}{l}\text { Fit into experimental } \\
\text { results in Fig. 9(a) }\end{array}$ \\
\hline$\alpha_{2}\left(K^{-1}\right)$ & Transverse direction CTE & $5.78 \times 10^{-7} \mathrm{~T}-5.75 \times 10^{-5}$ & Choy et al., 1981 \\
\hline$v_{12}$ & Poisson’s ratio & 0.465 & Measurement \\
\hline$v_{23}$ & Poisson’s ratio & 0.45 & Choy and Leung, 1985 \\
\hline$r^{0}(\mathrm{~mm})$ & $\begin{array}{l}\text { Radius for the inner most hollow } \\
\text { cylinder }\end{array}$ & $10^{-10}$ & $\begin{array}{l}\text { Suitable for simulation } \\
\text { of solid cylinder }\end{array}$ \\
\hline$r^{N}(m m)$ & $\begin{array}{l}\text { Radius for the outer most hollow } \\
\text { cylinder }\end{array}$ & 0.39 & Measurement \\
\hline \multicolumn{4}{|c|}{ Parameters for macro-scale modeling } \\
\hline$n$ & Number of active coils & 8 & Measurement \\
\hline$D(m m)$ & Outer diameter of the coil & 2.11 & Measurement \\
\hline$\alpha_{c}\left({ }^{\circ}\right)$ & Pitch angle & 10 & Measurement \\
\hline
\end{tabular}

\subsection{Comparison with experimental results}

\subsubsection{Axial modulus of Sample 1T}

The elastic modulus of the twisted fiber is reproduced using the meso-scale model and is presented in Fig. 8 (bottom dash line), together with the experiment results (bottom solid line). The modeling result exhibits good agreement with the experiment result. The small deviation of the modeling result from the experimental result at the beginning of the test is most likely attributed to the choice of the transverse elastic properties, which are not able to be obtained experimentally at this time.

\subsubsection{Torsional actuation of Sample $1 T$}

We used a torque sensor (Mark-10 MR50-50Z Plug \& Test Universal Torque Sensor, with a resolution of $1.41 \times 10^{-4} \mathrm{~N} \cdot \mathrm{m}$ ) to measure the torque generated by Sample $1 \mathrm{~T}$ fixed at both ends. Fig. 10 shows that our meso-scale model successfully captures the torsional actuation trend of the twisted fiber, which is essential to the tensile actuation behavior of the coiled spring. 


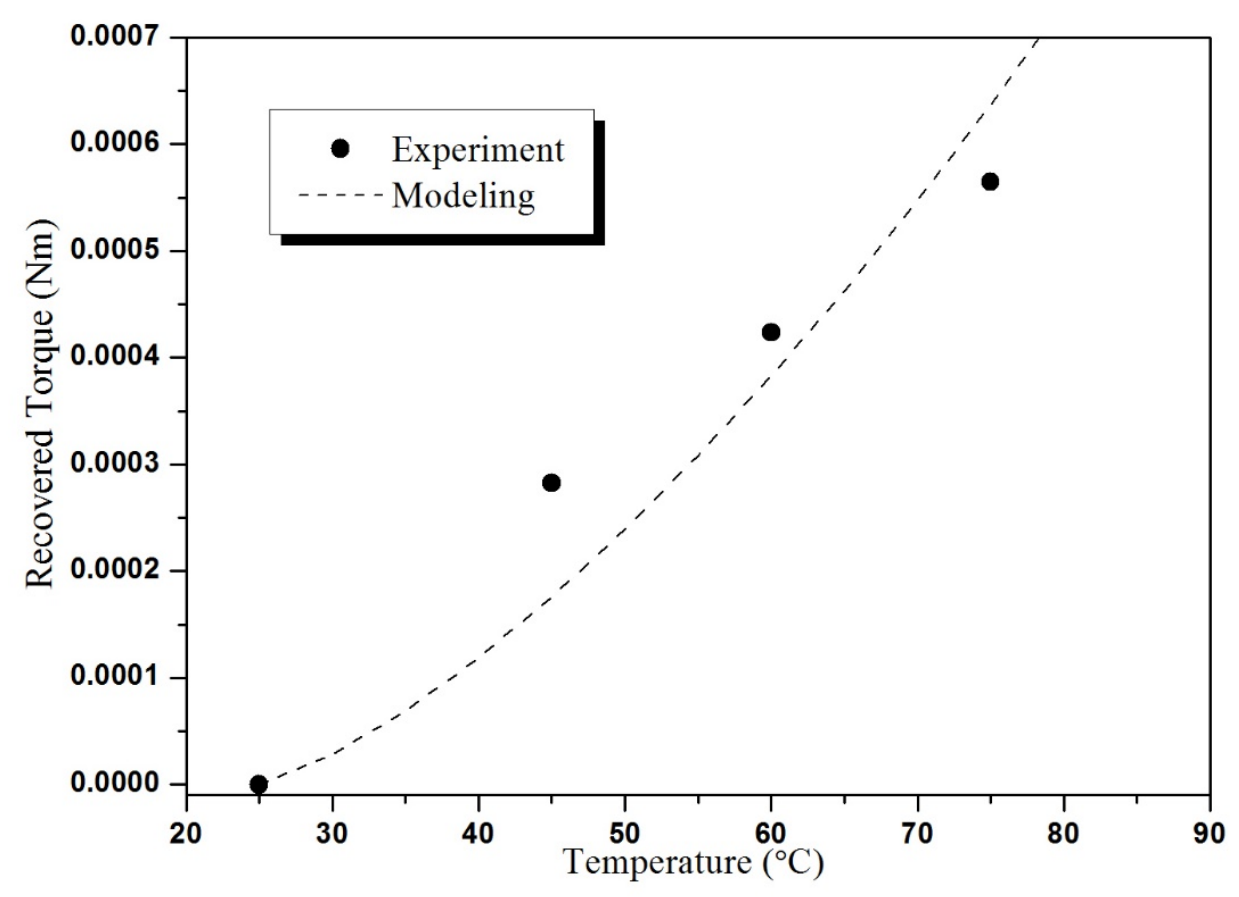

Fig. 10. Experiment and modeling results of the recovered torque as a function of temperature.

\subsubsection{Tensile actuation of Sample 2TC}

Finally, the tensile actuation of Sample 2TC is measured using DMA by applying a constant force of $1.5 \mathrm{~N}$ to the coiled spring, and heating and cooling the temperature between $25^{\circ} \mathrm{C}$ and $80^{\circ} \mathrm{C}$ at $10^{\circ} \mathrm{C} / \mathrm{min}$. Fig. 11 proves the ability of the macro-scale model in reproducing the trend of the coiled spring (muscle). 


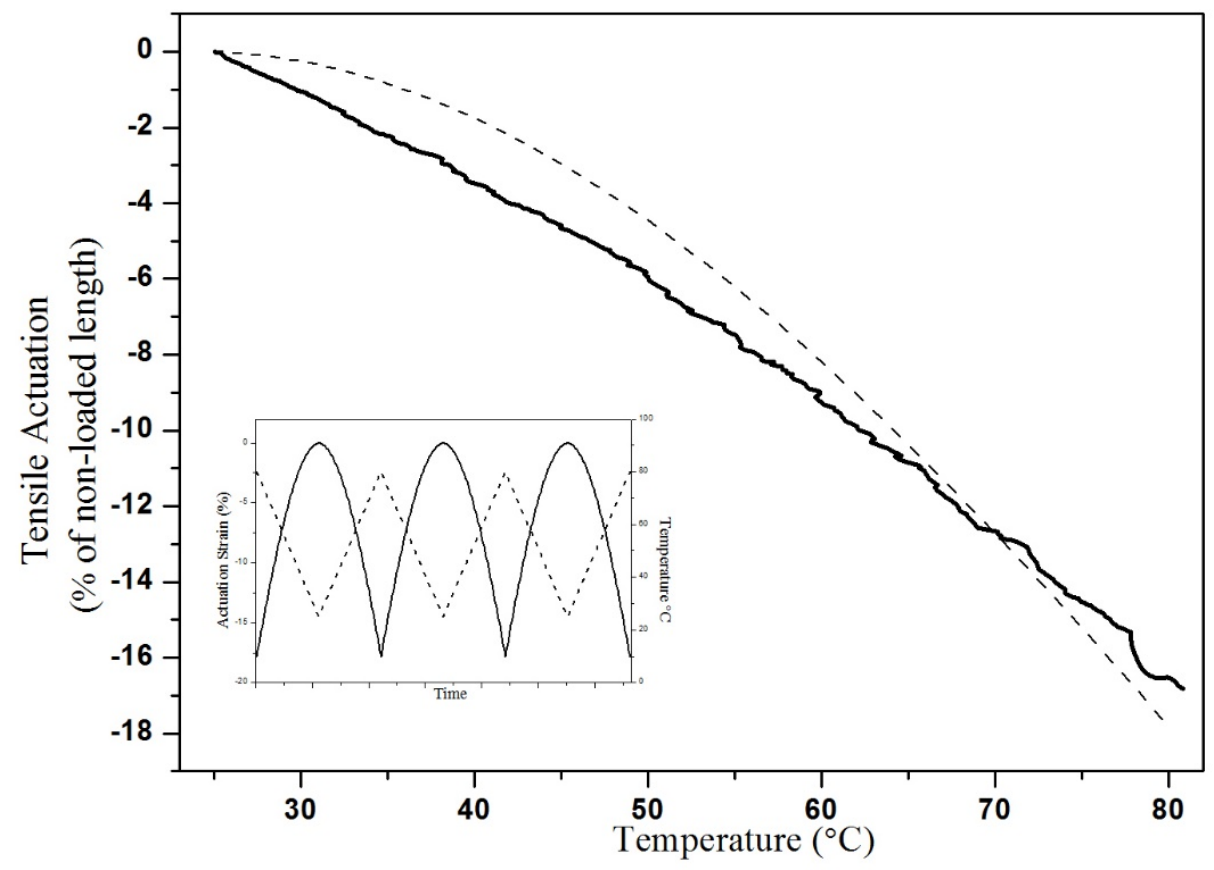

Fig. 11. Experiment and modeling results of the tensile actuation of the coiled spring (solid line: test results; dashed line: modeling results). The inset shows the simulation results of multiple actuation cycles (solid line: calculated results of strain actuation; dashed line: temperature).

\subsection{Prediction and Discussion}

To further demonstrate the prediction ability of our model, we here performed a series of parametric studies, aiming to investigate how the parameters at different scales affect the thermomechanical properties of the fiber, the torsional actuation response of the twisted fiber and eventually, the tensile actuation response of the coiled spring.

\subsubsection{Effect of micro-scale parameters on thermomechanical properties of the precursor fiber}

Fig. 12(a) exhibits the effect of crystalline micro-fibril volume fraction $\left(V^{f}\right)$ on the axial modulus of the precursor fiber. As expected, higher $V^{f}$ increases the Young's modulus of the precursor fiber in the axial direction. Likewise, the Young's modulus in the transverse direction and the shear modulus can also be increased by increasing $V^{f}$ (Not shown in the figure for brevity). 
One of the most efficient methods to enhance the mechanical properties of the asfabricated polymeric fibers is by highly stretching the fiber. During this procedure, the crystalline lamellae in the micro-fibrils are oriented along the draw direction and end up with crystalline micro-fibrils with higher aspect ratios $\left(l_{f} / r_{f}\right)$. In Fig. 12(b), we illustrate that higher aspect ratio of the micro-fibrils leads to higher axial modulus. It has also been experimentally demonstrated that the drawing procedure can produce higher thermal anisotropy, that is, higher thermal contraction in the axial direction and higher thermal expansion in the transverse direction (Choy et al., 1981). In the following subsection, we will show how the changes in thermomechanical properties of the precursor fiber affect the behavior of the twisted fiber.

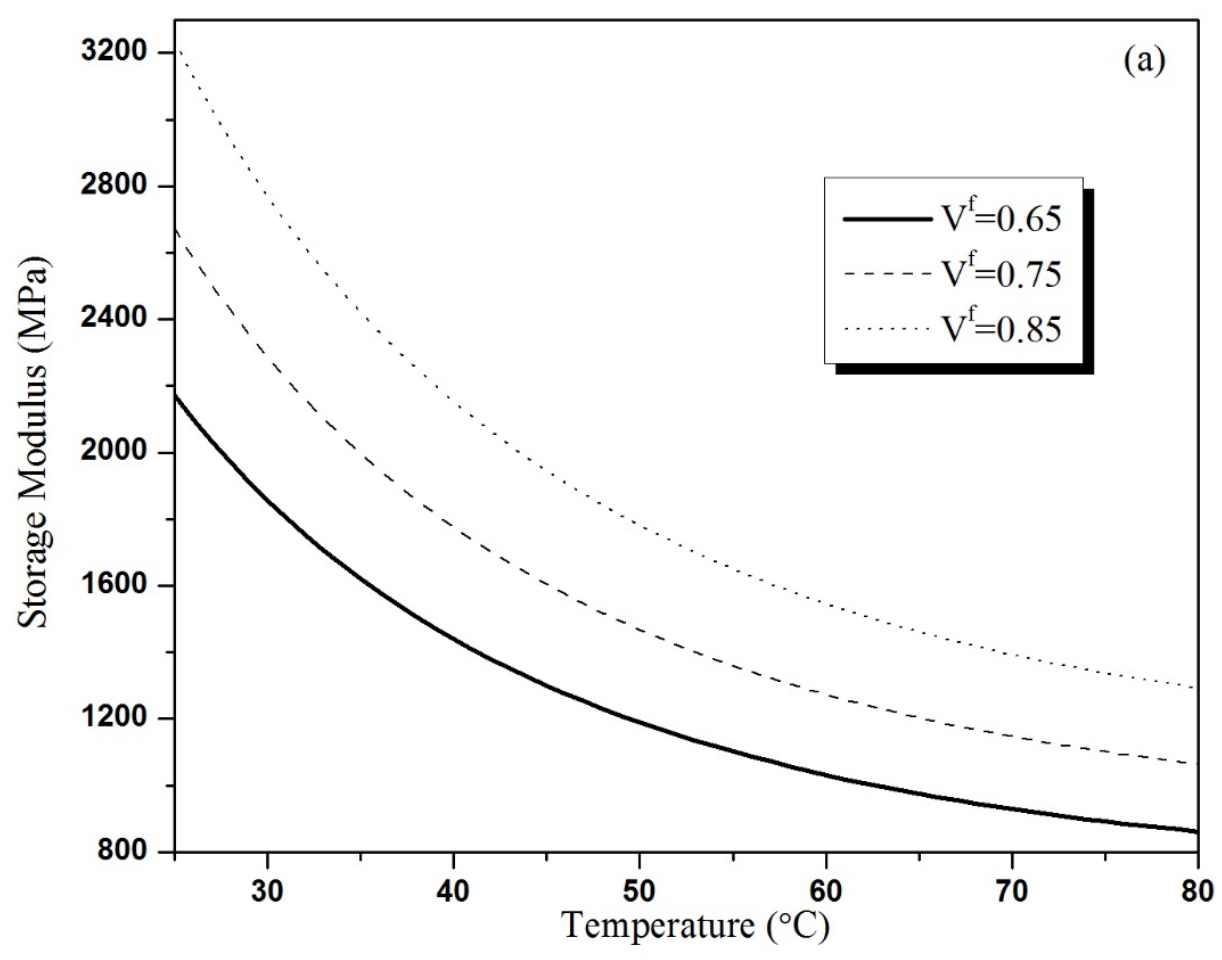




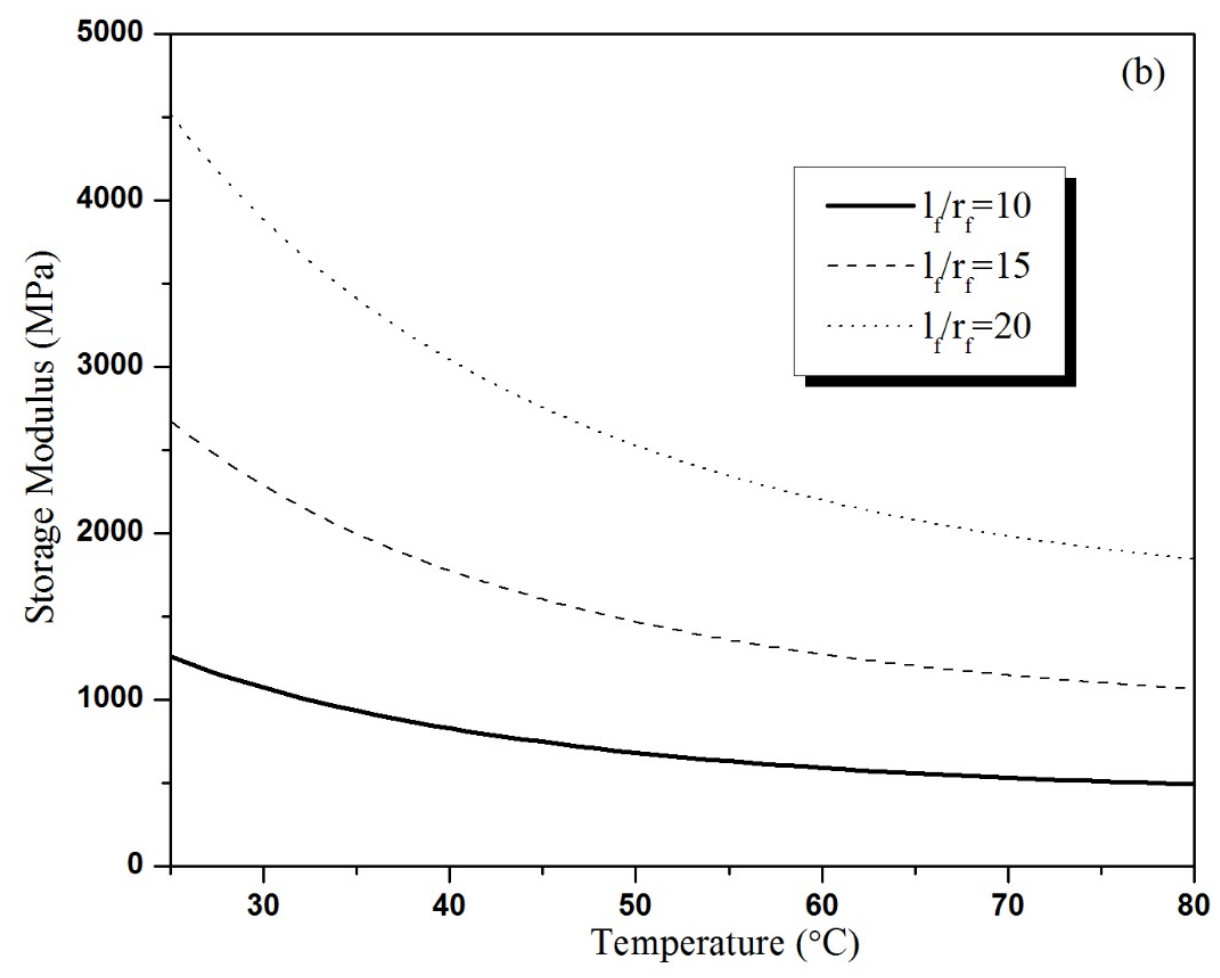

Fig. 12. Axial Young's modulus of the precursor fibers with different (a) crystalline micro-fibrils volume fractions and (b) micro-fibril aspect ratios.

\subsubsection{Effect of meso-scale parameters on torsional actuation response of the twisted fiber}

In Fig. 13(a), we compare the torsional behavior of the twisted fibers made of precursor fibers with different axial moduli, which is attributed to different micro-fibril aspect ratio. It is clear that the precursor fiber with higher micro-fibril aspect ratio has higher axial modulus (Fig. 12(b)), and eventually lead to better torsional actuation performance for the twisted fiber. In addition, based on a simple helix analysis, Haines et al. (2014) concluded that "both contraction of these helically configured polymer chains and fiber diameter expansion will cause fiber untwist". Therefore, it is envisioned that the larger the anisotropy between the thermal expansion in the axial and transverse direction, the higher the untwisting tendency, and hence the larger the recovered torque will be. This is proved in Fig. 13(b). Here we multiply the original CTE's of the twisted fiber "Anisotropy 1" by a factor of 2 and 3, resulting in twisted fiber specimens with larger anisotropies, specimen "Anisotropy 2" and specimen “Anisotropy 3", 
respectively. As stated above, a facile strategy to increase the thermal anisotropy is by stretching the precursor fiber (Choy et al., 1981).

According to the experimental results in Haines et al. (2014), the geometrical configurations of the twisted fibers also have a large impact on the actuation behavior. In Fig. 13(c), the recovered torque dependence on the twisted fiber bias angle is presented. It turns out that the torque does not follow a monotonous trend with respect to bias angles; instead, it reaches its maximum around $35^{\circ}$. The appearance of the maximum actuation is as a result of the various orientations in each cylindrical layer and the constraints between each layer (See Eq. A6d).

Predictions in Fig. 13 suggested that if we could find a fiber with a remarkable combination of high modulus and large thermal anisotropy, along with an appropriate fiber bias angle, which can be altered by applying different load during twisting insertion, the torsional actuation performance of the twisted fiber would be significantly improved. In the following subsection, we will show how the improvement in torsional actuation of the twisted fiber affect the manifestation of the coiled spring. 


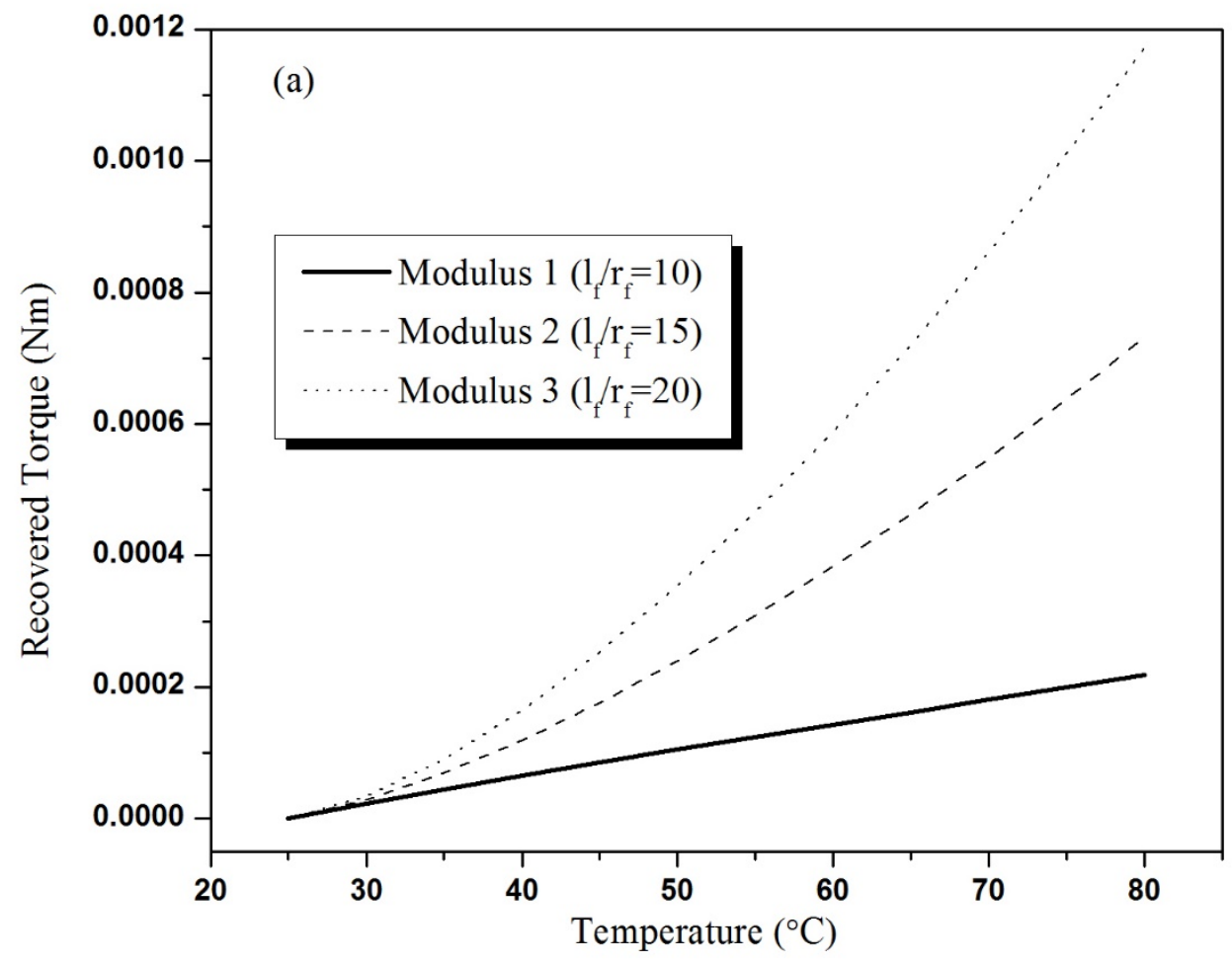



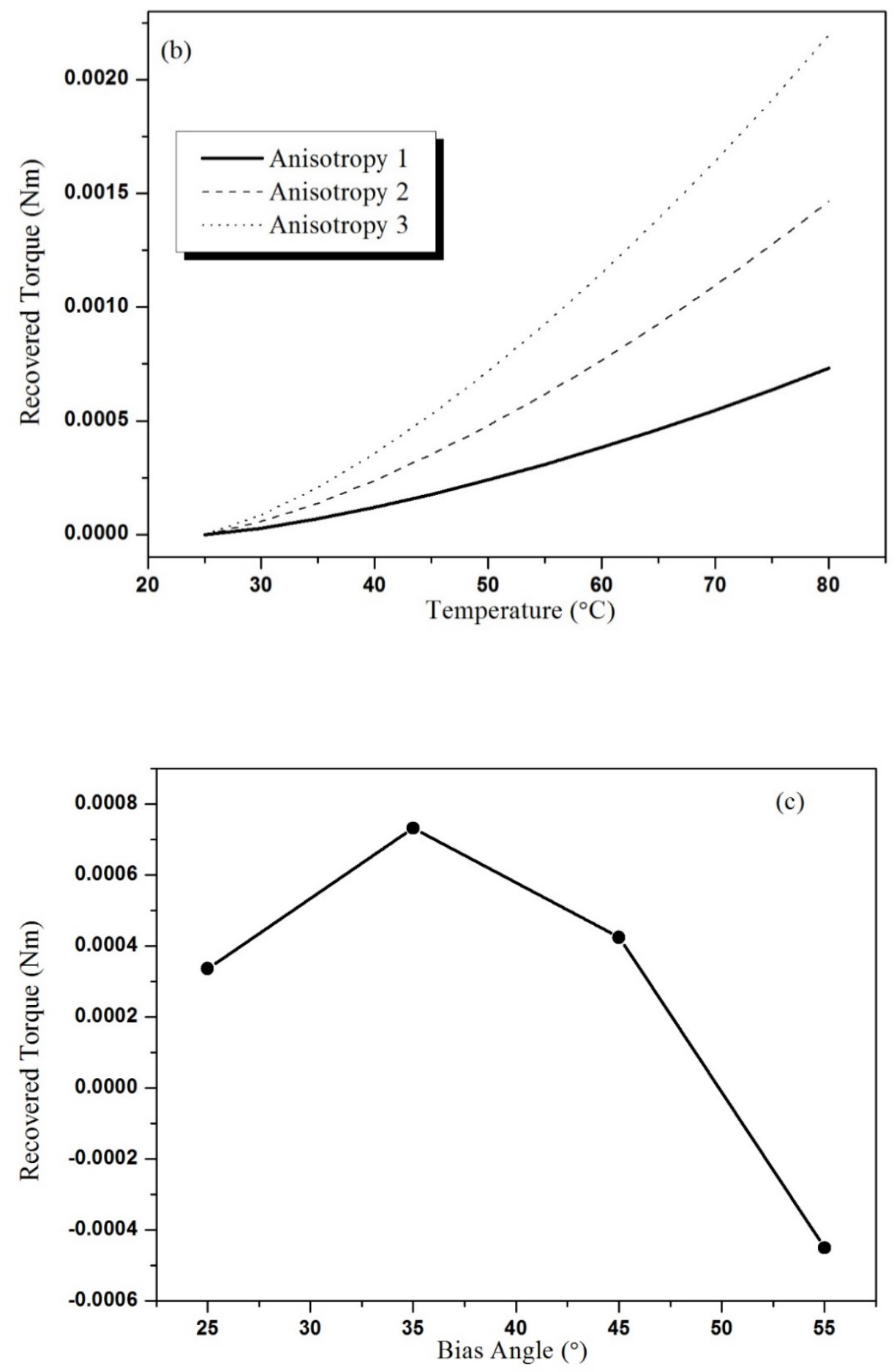

Fig. 13. Recovered torque generated by the twisted fiber with different (a) axial moduli, (b) thermal anisotropies, and (c) bias angles. 


\subsubsection{Effect of macro-scale parameters on tensile actuation response of the coiled spring}

As has been extensively stated in the previous sections, the remarkable tensile actuation behavior of the coiled spring is driven by the torsional actuation generated by the twisted fiber during heating. This is quantitatively reflected in Eq. (10), where it is clear that the external applied force $F$ is responsible for the elongation of the spring, just like in the regular spring with a stiffness of $f_{11}$. The recovered torque $M_{\text {rec }}$, however, depending on its sign, can either contribute to the total elongation (when twisting and coiling have opposite chirality and $M_{\text {rec }}$ is negative) or compensate for the external force and responsible for contraction of the spring (when twisting and coiling have the same chirality and $M_{\text {rec }}$ is positive). To clarify, we take the chirality of the coiled spring as the reference, i.e., the pitch angle of the coiled spring is always positive. In this way, the twisted fiber with chirality that matches that of the coiled spring has a positive fiber bias angle and this twisted-then-coiled spring is defined as a homochiral system. Likewise, the twisted fiber with chirality that is opposite to that of the coiled spring has a negative fiber bias angle and the resulting coiled spring is heterochiral. In Fig. 14(a), the effect of the fiber bias angle, and hence the recovered torque on the tensile actuation response of the artificial muscle is presented. When the fiber bias angle increases, more recovered torque can be generated upon heating, and thus results in better tensile actuation performance in both homochiral and heterochiral cases. As expected, the maximum stroke is again reached around $\pm 35^{\circ}$. Our prediction is very close to the experimental results in Fig. $4 \mathrm{~F}$ of Haines et al. (2014). Therefore, an appropriate fiber bias angle is an important factor to tune the tensile actuation performance. Without extensive experimental testing, our model provides an efficient numerical tool to seek the optimized fiber bias angle.

As pointed out in the previous section, the recovered torque of the twisted fiber, and hence the tensile actuation of the coiled spring, can also be increased by enhancing the axial modulus (Fig. 12(b) and Fig. 13(a)) and the thermal anisotropy (Fig. 13(b)) of the precursor fiber. One of the promising candidates could be two-way shape memory polymers (Behl et al., 2013a; Behl et al., 2013b; Chen et al., 2008; Chung et al., 2008; Hong et al., 2010; Lee et al., 2008; Li et al., 2011; Pandini et al., 2013; Pandini et al., 2015; Pandini et al., 2012; Raquez et al., 2011; Stroganov et al., 2015), which behave similarly to polymers with negative CTEs (i.e., heating induces contraction and cooling causes expansion). Because the two-way shape memory effect is 
much larger than the negative CTEs of the fishing lines, which suggests giant thermal anisotropy, it is expected that two-way shape memory polymers would have remarkable muscle behavior.

Finally, Fig. 14(b) exhibits the contracting stroke as a function of spring indices and shows that larger spring index leads to higher contracting stroke, which has also been proved experimentally in Haines et al. (2014) and Zhang and Li (2015). Nevertheless, smaller spring index may still be used in some applications when higher actuation force is in favor over higher stroke.

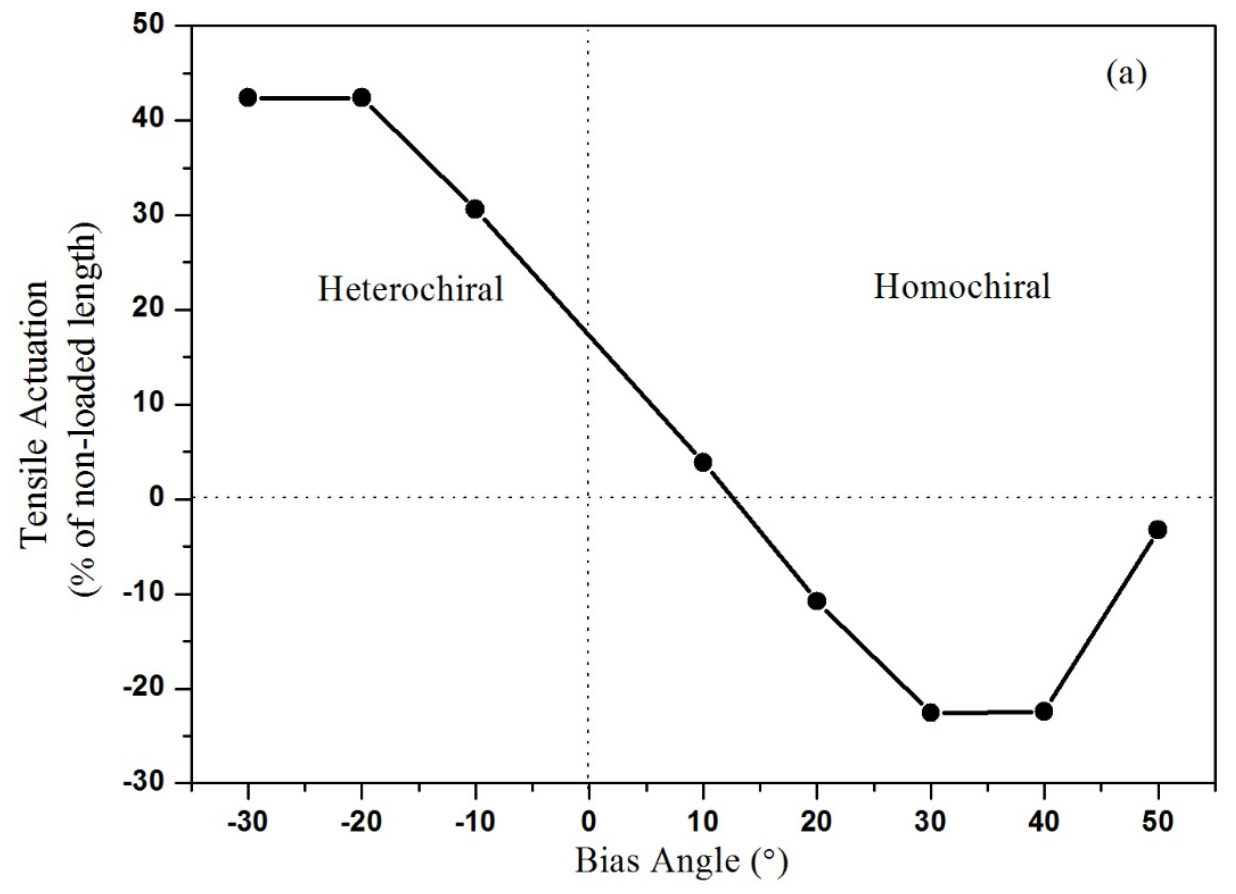




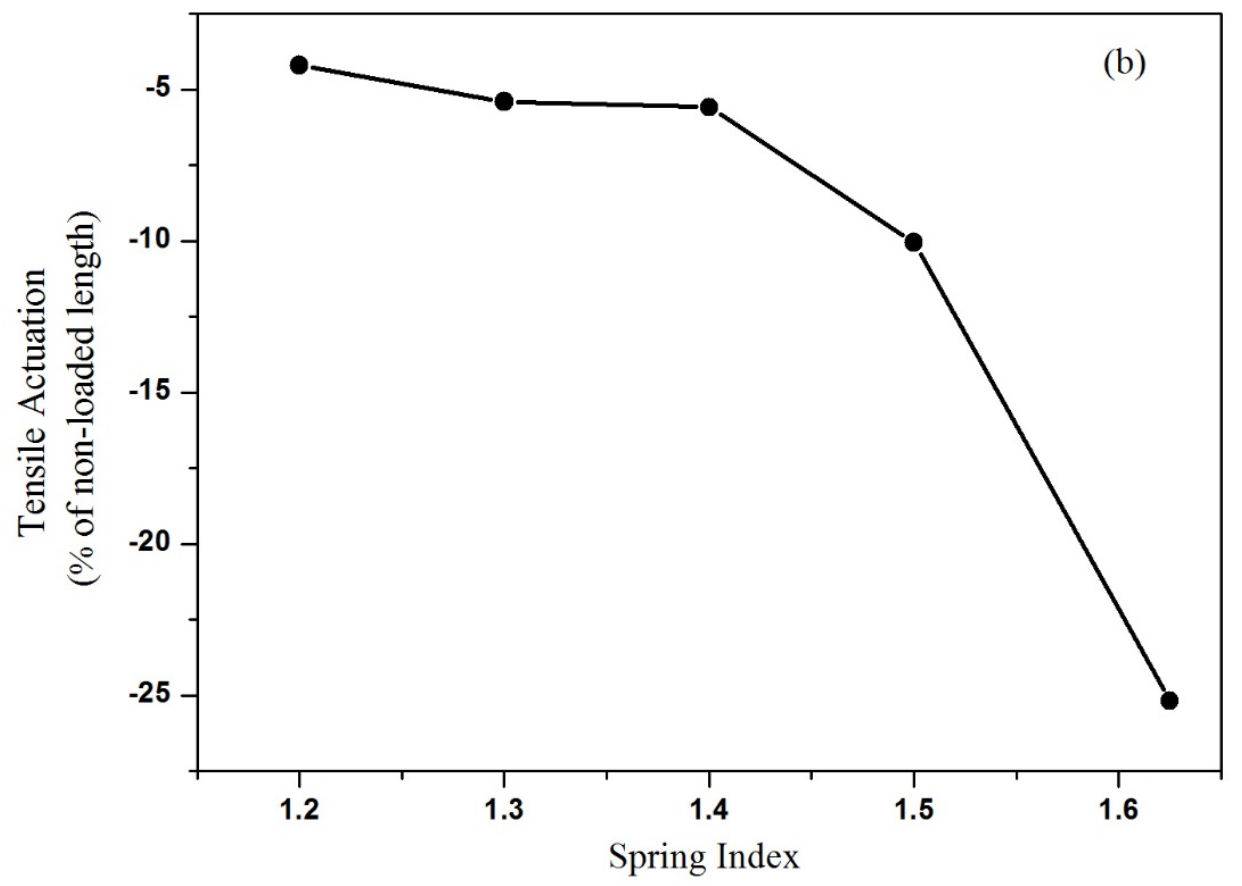

Fig. 14. Prediction of the tensile stroke as a function of (a) fiber bias angles and (b) spring indices.

\section{Conclusion}

In this paper, a multi-scale modeling framework based on a top-down analysis has been developed for the actuation response of the polymeric artificial muscles. The model was validated against the experimental results for the polyethylene copolymer fiber based muscles. The physical origin and dependence of the torsional actuation of the twisted fiber and the tensile actuation of the twisted-then-coiled spring on temperature have been elaborated and reproduced. A parametric study was further conducted to help future design and optimization of such innovative artificial muscles. The important results of this work include:

(1) In the macro-scale model, a consistent application of Castigliano's second theorem (CST) has been used, to account for the combined effect of the axial applied force and recovered torque on the thermally triggered tensile actuation. The combined contribution of the applied force and the recovered torque is evidenced by the fact that the tensile stroke is largely dependent on fiber bias angle, because the fiber bias angle plays an important role in 
determining the recovered torque. Another predication from our parametric study shows that the actuation behavior of the muscle can be tuned by spring indices of the twisted-then-coiled spring. More specifically, larger index results in higher tensile stroke.

(2) In the meso-scale model, the torsional behavior of the twisted fiber was reproduced by using classical composite material theory and helical anisotropic cylinder analysis. The appearance of a coupling term $\alpha_{z \theta}$ in the off-axis properties proves that a reversible thermal shear strain will be generated during heating or cooling. In other words, the torsional behavior of the twisted fiber can be explained from the composite material point of view. Parametric study indicates that there exists an optimized fiber bias angle that maximizes the recovered torque and hence the tensile stroke.

(3) The nano- and micro-scale modeling for the precursor fiber is material dependent. In our study, due to the fact that the morphology of the semi-crystalline polyethylene copolymer fiber consists of discontinuous crystalline micro-fibrils embedded into an amorphous matrix, we make use of the classical theories in continuum mechanics to simulate the thermomechanical properties of the fiber. Parametric studies suggest that by increasing the modulus and the thermal anisotropy of the precursor fiber, the actuation performance of the artificial muscle will be greatly improved.

(4) Some new thermally reversible polymers, such as two-way shape memory polymers, may provide remarkable actuation response.

\section{Acknowledgement}

This study was financially supported by National Science Foundation under grant number CMMI 1333997. The authors also gratefully appreciate the help from Dr. Pengfei Zhang for the experimental tests. 


\section{Appendix A: Analysis of Cylindrical Laminate}

By assuming axisymmetric radial deformation and shearing twist, and axial extension that is uniform across the cross-section, the displacements are shown to be functions of the radial and axial coordinates (See Fig. 4):

$$
u=u(r) \quad v=v_{0} r z \quad w=w_{0} z
$$

The corresponding strain components are:

$$
\varepsilon_{r}=\frac{d u}{d r} \quad \varepsilon_{\theta}=\frac{u}{r} \quad \varepsilon_{z}=w_{0} \quad \varepsilon_{z \theta}=v_{0} r \quad \varepsilon_{r \theta}=\varepsilon_{r z}=0
$$

By assuming infinitesimal deformation of the material, the constitutive relation for a material with helical anisotropy for the four non-zero stress and strain components is:

$$
\left[\begin{array}{c}
\sigma_{z} \\
\sigma_{\theta} \\
\sigma_{r} \\
\sigma_{z \theta}
\end{array}\right]=\left[\begin{array}{llll}
\bar{C}_{11} & \bar{C}_{12} & \bar{C}_{13} & \bar{C}_{16} \\
\bar{C}_{12} & \bar{C}_{22} & \bar{C}_{23} & \bar{C}_{26} \\
\bar{C}_{13} & \bar{C}_{23} & \bar{C}_{33} & \bar{C}_{36} \\
\bar{C}_{16} & \bar{C}_{26} & \bar{C}_{36} & \bar{C}_{66}
\end{array}\right]\left[\begin{array}{c}
\varepsilon_{z}-\alpha_{z} \Delta T \\
\varepsilon_{\theta}-\alpha_{\theta} \Delta T \\
\varepsilon_{r}-\alpha_{r} \Delta T \\
\varepsilon_{z \theta}-\alpha_{z \theta} \Delta T
\end{array}\right]
$$

The equilibrium equation that needs to be satisfied is:

$$
\frac{d \sigma_{r}}{d r}+\frac{\sigma_{r}-\sigma_{\theta}}{r}=0
$$

Substituting Eqs. (A.1), (A.2) and (A.3) into the governing equation (A.4) leads to the radial displacement $u$. Without going to further derivation details, we here present the solution for $u$ :

$$
\begin{aligned}
& u=C_{1} r^{\mu}+C_{2} r^{-\mu}+\frac{\left(\bar{C}_{26}-2 \bar{C}_{36}\right)}{\left(4 \bar{C}_{33}-\bar{C}_{22}\right)} v_{0} r^{2} \\
& +\frac{\left(\bar{C}_{12}-\bar{C}_{13}\right)}{\left(\bar{C}_{33}-\bar{C}_{22}\right)} w_{0} r+\frac{\left(\bar{C}_{13}-\bar{C}_{12}\right)}{\left(\bar{C}_{33}-\bar{C}_{22}\right)} \alpha_{z} \Delta \operatorname{Tr}+\frac{\left(\bar{C}_{23}-\bar{C}_{22}\right)}{\left(\bar{C}_{33}-\bar{C}_{22}\right)} \alpha_{\theta} \Delta \operatorname{Tr} \\
& +\frac{\left(\bar{C}_{33}-\bar{C}_{23}\right)}{\left(\bar{C}_{33}-\bar{C}_{22}\right)} \alpha_{r} \Delta \operatorname{Tr}+\frac{\left(\bar{C}_{36}-\bar{C}_{26}\right)}{\left(\bar{C}_{33}-\bar{C}_{22}\right)} \alpha_{z \theta} \Delta \operatorname{Tr}
\end{aligned}
$$

where $\mu=\sqrt{\frac{\bar{C}_{22}}{\bar{C}_{33}}}$, and the undetermined coefficients $C_{1}$ and $C_{2}$ will be determined by the continuity and boundary conditions as shown in Sub-section 3.2.2. The stress components can then be obtained by taking Eq. (A.5) back into Eqs. (A.1), (A.2) and (A.3): 


$$
\begin{aligned}
& \sigma_{z}=C_{1} r^{\mu-1}\left(\bar{C}_{12}+\mu \bar{C}_{13}\right)+C_{2} r^{-\mu-1}\left(\bar{C}_{12}-\mu \bar{C}_{13}\right) \\
& +w_{0}\left[\bar{C}_{11}+\frac{\left(\bar{C}_{12}+\bar{C}_{13}\right)\left(\bar{C}_{12}-\bar{C}_{13}\right)}{\left(\bar{C}_{33}-\bar{C}_{22}\right)}\right] \\
& +v_{0} r\left[\bar{C}_{16}+\frac{\left(\bar{C}_{12}+2 \bar{C}_{13}\right)\left(\bar{C}_{26}-2 \bar{C}_{36}\right)}{\left(4 \bar{C}_{33}-\bar{C}_{22}\right)}\right] \\
& +\alpha_{z} \Delta T\left[-\bar{C}_{11}+\frac{\left(\bar{C}_{12}+\bar{C}_{13}\right)\left(\bar{C}_{13}-\bar{C}_{12}\right)}{\left(\bar{C}_{33}-\bar{C}_{22}\right)}\right] \\
& +\alpha_{\theta} \Delta T\left[-\bar{C}_{12}+\frac{\left(\bar{C}_{12}+\bar{C}_{13}\right)\left(\bar{C}_{23}-\bar{C}_{22}\right)}{\left(\bar{C}_{33}-\bar{C}_{22}\right)}\right] \\
& +\alpha_{r} \Delta T\left[-\bar{C}_{13}+\frac{\left(\bar{C}_{12}+\bar{C}_{13}\right)\left(\bar{C}_{33}-\bar{C}_{23}\right)}{\left(\bar{C}_{33}-\bar{C}_{22}\right)}\right] \\
& +\alpha_{z \theta} \Delta T\left[-\bar{C}_{16}+\frac{\left(\bar{C}_{12}+\bar{C}_{13}\right)\left(\bar{C}_{36}-\bar{C}_{26}\right)}{\left(\bar{C}_{33}-\bar{C}_{22}\right)}\right] \\
& \sigma_{\theta}=C_{1} r^{\mu-1}\left(\bar{C}_{22}+\mu \bar{C}_{23}\right)+C_{2} r^{-\mu-1}\left(\bar{C}_{22}-\mu \bar{C}_{23}\right) \\
& +w_{0}\left[\bar{C}_{12}+\frac{\left(\bar{C}_{22}+\bar{C}_{23}\right)\left(\bar{C}_{12}-\bar{C}_{13}\right)}{\left(\bar{C}_{33}-\bar{C}_{22}\right)}\right] \\
& +v_{0} r\left[\bar{C}_{26}+\frac{\left(\bar{C}_{22}+2 \bar{C}_{23}\right)\left(\bar{C}_{26}-2 \bar{C}_{36}\right)}{\left(4 \bar{C}_{33}-\bar{C}_{22}\right)}\right] \\
& +\alpha_{z} \Delta T\left[-\bar{C}_{12}+\frac{\left(\bar{C}_{22}+\bar{C}_{23}\right)\left(\bar{C}_{13}-\bar{C}_{12}\right)}{\left(\bar{C}_{33}-\bar{C}_{22}\right)}\right] \\
& +\alpha_{\theta} \Delta T\left[-\bar{C}_{22}+\frac{\left(\bar{C}_{22}+\bar{C}_{23}\right)\left(\bar{C}_{23}-\bar{C}_{22}\right)}{\left(\bar{C}_{33}-\bar{C}_{22}\right)}\right] \\
& +\alpha_{r} \Delta T\left[-\bar{C}_{23}+\frac{\left(\bar{C}_{22}+\bar{C}_{23}\right)\left(\bar{C}_{33}-\bar{C}_{23}\right)}{\left(\bar{C}_{33}-\bar{C}_{22}\right)}\right] \\
& +\alpha_{z \theta} \Delta T\left[-\bar{C}_{26}+\frac{\left(\bar{C}_{22}+\bar{C}_{23}\right)\left(\bar{C}_{36}-\bar{C}_{26}\right)}{\left(\bar{C}_{33}-\bar{C}_{22}\right)}\right] \\
& \sigma_{r}=C_{1} r^{\mu-1}\left(\bar{C}_{23}+\mu \bar{C}_{33}\right)+C_{2} r^{-\mu-1}\left(\bar{C}_{23}-\mu \bar{C}_{33}\right) \\
& +w_{0}\left[\bar{C}_{13}+\frac{\left(\bar{C}_{23}+\bar{C}_{33}\right)\left(\bar{C}_{12}-\bar{C}_{13}\right)}{\left(\bar{C}_{33}-\bar{C}_{22}\right)}\right]
\end{aligned}
$$




$$
\begin{aligned}
& +v_{0} r\left[\bar{C}_{36}+\frac{\left(\bar{C}_{23}+2 \bar{C}_{33}\right)\left(\bar{C}_{26}-2 \bar{C}_{36}\right)}{\left(4 \bar{C}_{33}-\bar{C}_{22}\right)}\right] \\
& +\alpha_{z} \Delta T\left[-\bar{C}_{13}+\frac{\left(\bar{C}_{23}+\bar{C}_{33}\right)\left(\bar{C}_{13}-\bar{C}_{12}\right)}{\left(\bar{C}_{33}-\bar{C}_{22}\right)}\right] \\
& +\alpha_{\theta} \Delta T\left[-\bar{C}_{23}+\frac{\left(\bar{C}_{23}+\bar{C}_{33}\right)\left(\bar{C}_{23}-\bar{C}_{22}\right)}{\left(\bar{C}_{33}-\bar{C}_{22}\right)}\right] \\
& +\alpha_{r} \Delta T\left[-\bar{C}_{33}+\frac{\left(\bar{C}_{23}+\bar{C}_{33}\right)\left(\bar{C}_{33}-\bar{C}_{23}\right)}{\left(\bar{C}_{33}-\bar{C}_{22}\right)}\right] \\
& +\alpha_{z \theta} \Delta T\left[-\bar{C}_{36}+\frac{\left(\bar{C}_{23}+\bar{C}_{33}\right)\left(\bar{C}_{36}-\bar{C}_{26}\right)}{\left(\bar{C}_{33}-\bar{C}_{22}\right)}\right]
\end{aligned}
$$

$$
\sigma_{\theta z}=C_{1} r^{\mu-1}\left(\bar{C}_{26}+\mu \bar{C}_{26}\right)+C_{2} r^{-\mu-1}\left(\bar{C}_{26}-\mu \bar{C}_{26}\right)
$$$$
+w_{0}\left[\bar{C}_{16}+\frac{\left(\bar{C}_{26}+\bar{C}_{36}\right)\left(\bar{C}_{12}-\bar{C}_{13}\right)}{\left(\bar{C}_{33}-\bar{C}_{22}\right)}\right]
$$$$
+v_{0} r\left[\bar{C}_{66}+\frac{\left(\bar{C}_{26}+2 \bar{C}_{36}\right)\left(\bar{C}_{26}-2 \bar{C}_{36}\right)}{\left(4 \bar{C}_{33}-\bar{C}_{22}\right)}\right]
$$$$
+\alpha_{z} \Delta T\left[-\bar{C}_{16}+\frac{\left(\bar{C}_{26}+\bar{C}_{36}\right)\left(\bar{C}_{13}-\bar{C}_{12}\right)}{\left(\bar{C}_{33}-\bar{C}_{22}\right)}\right]
$$$$
+\alpha_{\theta} \Delta T\left[-\bar{C}_{26}+\frac{\left(\bar{C}_{26}+\bar{C}_{36}\right)\left(\bar{C}_{23}-\bar{C}_{22}\right)}{\left(\bar{C}_{33}-\bar{C}_{22}\right)}\right]
$$$$
+\alpha_{r} \Delta T\left[-\bar{C}_{36}+\frac{\left(\bar{C}_{26}+\bar{C}_{36}\right)\left(\bar{C}_{33}-\bar{C}_{23}\right)}{\left(\bar{C}_{33}-\bar{C}_{22}\right)}\right]
$$

$+\alpha_{z \theta} \Delta T\left[-\bar{C}_{66}+\frac{\left(\bar{C}_{26}+\bar{C}_{36}\right)\left(\bar{C}_{36}-\bar{C}_{26}\right)}{\left(\bar{C}_{33}-\bar{C}_{22}\right)}\right]$ 


\section{Appendix B: Flowchart of Simulation with the MATLAB Program}

The simulation flowchart using the MATLAB program is shown in Fig. B1.

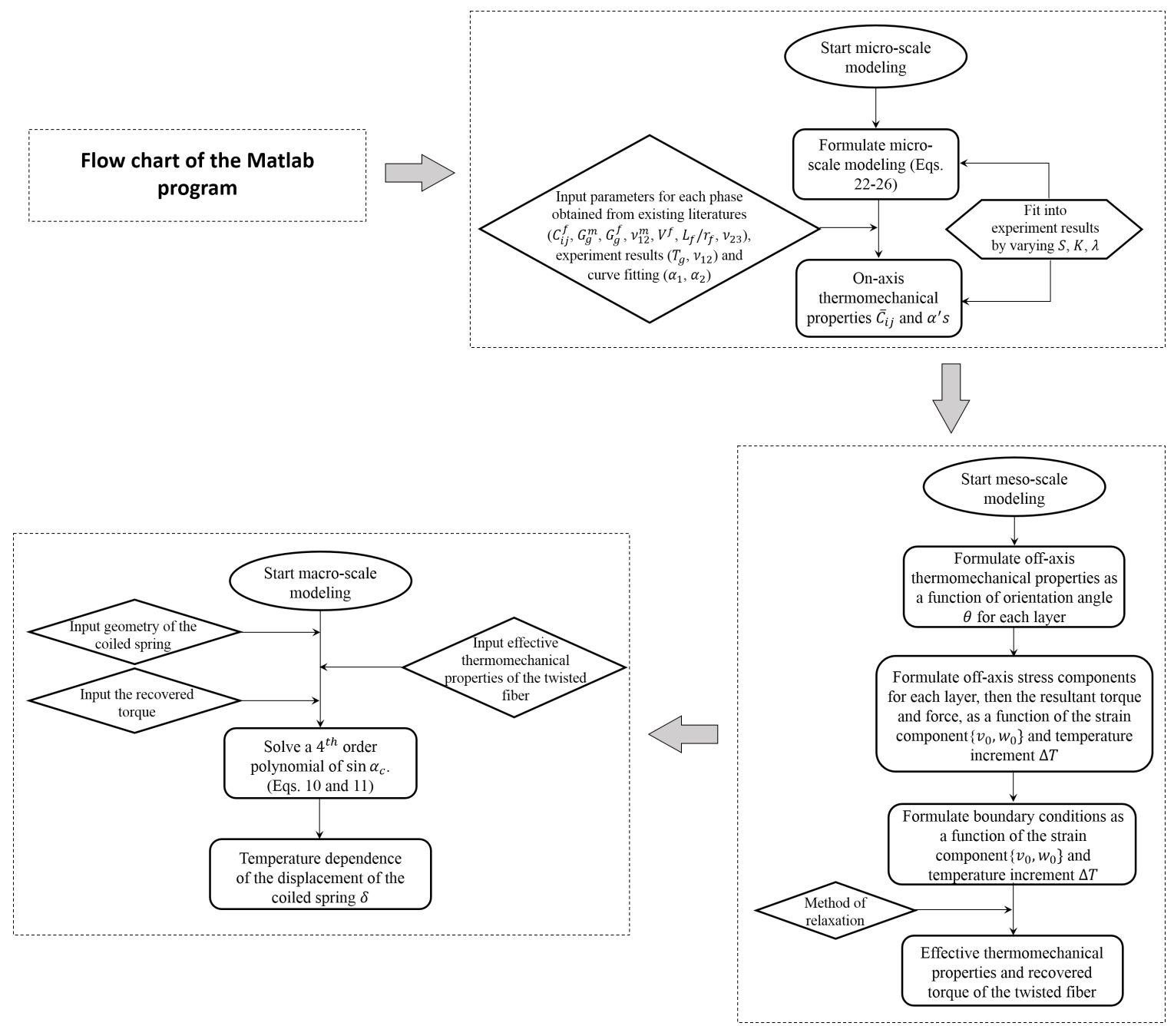

Fig. B1. Flow chart of the Matlab program for the multi-scale modeling. 


\section{References}

Aziz, S., Naficy, S., Foroughi, J., Brown, H.R., Spinks, G.M., 2015. Characterisation of torsional actuation in highly twisted yarns and fibres. Polym. Test. 46, 88-97.

Barham, P., Arridge, R., 1977. A fiber composite model of highly oriented polyethylene. J. Polym. Sci., Part B: Polym. Phys. 15, 1177-1188.

Baughman, R., 1996. Conducting polymer artificial muscles. Synth. Met. 78, 339-353.

Baughman, R.H., 1973. Negative thermal expansion in crystalline linear polymers. J. Chem. Phys. 58, 2976-2983.

Behl, M., Kratz, K., Noechel, U., Sauter, T., Lendlein, A., 2013a. Temperature-memory polymer actuators. Proc. Natl. Acad. Sci. U.S.A. 110, 12555-12559.

Behl, M., Kratz, K., Zotzmann, J., Nöchel, U., Lendlein, A., 2013b. Reversible Bidirectional Shape-Memory Polymers. Adv. Mater. 25, 4466-4469.

Boyd, R.H., 1979. The modulus of the amorphous component in polyethylenes. Polym. Eng. Sci. 19, 1010-1016.

Chan, O., Chen, F., Choy, C., Ward, I., 1978. The elastic constants of extruded polypropylene and polyethylene terephthalate. J. Phys. D: Appl. Phys. 11, 617.

Chen, S., Hu, J., Zhuo, H., Zhu, Y., 2008. Two-way shape memory effect in polymer laminates. Mater. Lett. 62, 4088-4090.

Cherubini, A., Moretti, G., Vertechy, R., Fontana, M., 2015. Experimental characterization of thermally-activated artificial muscles based on coiled nylon fishing lines. AIP Adv. 5, 067158.

Chou, C.-P., Hannaford, B., 1996. Measurement and modeling of McKibben pneumatic artificial muscles. IEEE Trans. Robot. Autom. 12, 90-102.

Choy, C., Chen, F., Ong, E., 1979. Anistropic thermal expansion of oriented crystalline polymers. Polym. 20, 1191-1198.

Choy, C., Chen, F., Young, K., 1981. Negative thermal expansion in oriented crystalline polymers. J. Polym. Sci., Part B: Polym. Phys. 19, 335-352.

Choy, C., Leung, W., 1985. Elastic moduli of ultradrawn polyethylene. J. Polym. Sci., Polym. Phy. Ed. 23, 1759-1780.

Chung, T., Romo-Uribe, A., Mather, P.T., 2008. Two-way reversible shape memory in a semicrystalline network. Macromolecules 41, 184-192. 
Clive, D., Shames, I., 1973. Solid Mechanics: A Variational Approach. McGraw-Hill, New York. Cox, H., 1952. The elasticity and strength of paper and other fibrous materials. Br. J. Appl. Phys. $3,72$.

Daerden, F., Lefeber, D., 2002. Pneumatic artificial muscles: actuators for robotics and automation. Europ. J. Mech. Envir. Eng. 47, 11-21.

Davis, G., Eby, R., Colson, J., 1970. Thermal expansion of polyethylene unit cell: effect of lamella thickness. J. Appl. Phys. 41, 4316-4326.

Dym, C.L., 1997. Structural modeling and analysis. Cambridge University Press, New York.

Dym, C.L., 2009. Consistent derivations of spring rates for helical springs. J. Mech. Design 131, 071004.

Foroughi, J., Spinks, G.M., Wallace, G.G., Oh, J., Kozlov, M.E., Fang, S., Mirfakhrai, T., Madden, J.D., Shin, M.K., Kim, S.J., 2011. Torsional carbon nanotube artificial muscles. Science 334, 494-497.

Grubb, D.T., Prasad, K., 1992. High-modulus polyethylene fiber structure as shown by x-ray diffraction. Macromolecules 25, 4575-4582.

Guo, X., Liu, L., Zhou, B., Liu, Y., Leng, J., 2015. Constitutive model for shape memory polymer based on the viscoelasticity and phase transition theories. J. Intell. Mater. Syst. Struct., 1045389X15571380.

Haines, C.S., Lima, M.D., Li, N., Spinks, G.M., Foroughi, J., Madden, J.D., Kim, S.H., Fang, S., de Andrade, M.J., Göktepe, F., 2014. Artificial muscles from fishing line and sewing thread. Science 343, 868-872.

Halpin, J.C., 1969. Effects of Environmental Factors on Composite Materials. DTIC Document.

Halpin, J.C., Kardos, J., 1976. The Halpin-Tsai equations: a review. Polym. Eng. Sci. 16, 344352.

He, T., 1986. An estimate of the strength of polymers. Polym. 27, 253-255.

Holliday, L., White, J., 1971. The stiffness of polymers in relation to their structure. Pure Appl. Chem. 26, 545-582.

Hong, S.J., Yu, W.-R., Youk, J.H., 2010. Two-way shape memory behavior of shape memory polyurethanes with a bias load. Smart Mater. Struct. 19, 035022. 
Kip, B.J., Van Eijk, M.C., Meier, R.J., 1991. Molecular deformation of high - modulus polyethylene fibers studied by micro - raman spectroscopy. J. Polym. Sci., Part B: Polym. Phys. 29, 99-108.

Klei, H.E., Stewart, J.J., 1986. Calculation of polymer elastic moduli using semiempirical methods. Int. J. Quantum Chem 30, 529-540.

Kobayashi, Y., Keller, A., 1970. The temperature coefficient of the c lattice parameter of polyethylene; an example of thermal shrinkage along the chain direction. Polym. 11, 114117.

Lee, K.M., Knight, P.T., Chung, T., Mather, P.T., 2008. Polycaprolactone- POSS Chemical/Physical Double Networks. Macromolecules 41, 4730-4738.

Leng, J., Lan, X., Liu, Y., Du, S., 2011. Shape-memory polymers and their composites: stimulus methods and applications. Prog. Mater Sci. 56, 1077-1135.

Li, J., Rodgers, W.R., Xie, T., 2011. Semi-crystalline two-way shape memory elastomer. Polym. 52, 5320-5325.

Lima, M.D., Li, N., De Andrade, M.J., Fang, S., Oh, J., Spinks, G.M., Kozlov, M.E., Haines, C.S., Suh, D., Foroughi, J., 2012. Electrically, chemically, and photonically powered torsional and tensile actuation of hybrid carbon nanotube yarn muscles. Science 338, 928932.

Love, A., 1944. A Treatise on the Mathematical theory of Elasticity. Dover Publication, New York.

Ma, H., Li, Y., Wang, D., 2014. Investigations of fiber twist on the mechanical properties of sisal fiber yarns and their composites. J. Reinf. Plast. Compos. DOI: 10.1177/0731684413520187.

Madden, J.D., Vandesteeg, N., Anquetil, P., Madden, P.G., Takshi, A., Pytel, R.Z., Lafontaine, S.R., Wieringa, P., Hunter, I.W., 2004. Artificial muscle technology: physical principles and naval prospects. IEEE J. Ocean. Eng. 29, 706-728.

Maranganti, R., Sharma, P., 2007. Length scales at which classical elasticity breaks down for various materials. Phys. Rev. Lett. 98, 195504.

McEvoy, M., Correll, N., 2015. Materials that couple sensing, actuation, computation, and communication. Science 347, 1261689. 
Militký, J., and Kovačič, V., 2011. Twist level influence on the dynamic mechanical characteristics of polyamide filament. Proc. Higher Ed. Inst., 55.

Mirvakili, S.M., Pazukha, A., Sikkema, W., Sinclair, C.W., Spinks, G.M., Baughman, R.H., Madden, J.D., 2013. Niobium nanowire yarns and their application as artificial muscles. Adv. Funct. Mater. 23, 4311-4316.

Moretti, G., Cherubini, A., Vertechy, R., Fontana, M., 2015. Experimental characterization of a new class of polymeric-wire coiled transducers, SPIE Smart Structures and Materials+Nondestructive Evaluation and Health Monitoring. International Society for Optics and Photonics, pp. 94320P-94320P.

Odajima, A., Maeda, T., 1967. Calculation of the elastic constants and the lattice energy of the polyethylene crystal, J. Polym. Sci., Part C: Polym. Sym. Wiley Online Library, pp. 5574.

Ohm, C., Brehmer, M., Zentel, R., 2010. Liquid crystalline elastomers as actuators and sensors. Adv. Mater. 22, 3366-3387.

Pandini, S., Baldi, F., Paderni, K., Messori, M., Toselli, M., Pilati, F., Gianoncelli, A., Brisotto, M., Bontempi, E., Ricco, T., 2013. One-way and two-way shape memory behaviour of semi-crystalline networks based on sol-gel cross-linked poly ( $\varepsilon$-caprolactone). Polym. 54, 4253-4265.

Pandini, S., Dioni, D., Paderni, K., Messori, M., Toselli, M., Bontempi, E., Riccò, T., 2015. The two-way shape memory behaviour of crosslinked poly ( $\varepsilon$-caprolactone) systems with largely varied network density. J. Intell. Mater. Syst. Struct., $1045389 X 15591384$.

Pandini, S., Passera, S., Messori, M., Paderni, K., Toselli, M., Gianoncelli, A., Bontempi, E., Ricco, T., 2012. Two-way reversible shape memory behaviour of crosslinked poly $(\varepsilon-$ caprolactone). Polym. 53, 1915-1924.

Pechhold, W., Blasenbrey, S., 1970. Molekülbewegung in Polymeren. Colloid Polym. Sci. 241, 955-976.

Pelrine, R., Kornbluh, R.D., Pei, Q., Stanford, S., Oh, S., Eckerle, J., Full, R.J., Rosenthal, M.A., Meijer, K., 2002. Dielectric elastomer artificial muscle actuators: toward biomimetic motion, SPIE's 9th Annual International Symposium on Smart Structures and Materials. International Society for Optics and Photonics, pp. 126-137. 
Pipes, R.B., Hubert, P., 2002. Helical carbon nanotube arrays: mechanical properties. Compos. Sci. Technol. 62, 419-428.

Pipes, R.B., Hubert, P., 2003. Helical carbon nanotube arrays: thermal expansion. Compos. Sci. Technol. 63, 1571-1579.

Raquez, J.M., Vanderstappen, S., Meyer, F., Verge, P., Alexandre, M., Thomassin, J.M., Jérôme, C., Dubois, P., 2011. Design of Cross - Linked Semicrystalline Poly ( $\varepsilon$ caprolactone) - Based Networks with One - Way and Two - Way Shape - Memory Properties through Diels-Alder Reactions. Chem. Eur. J. 17, 10135-10143.

Rider, J., Watkinson, K., 1978. Ultrasonic measurements of the elastic stiffness constants of oriented polyethylene. Polym. 19, 645-653.

Shahinpoor, M., Bar-Cohen, Y., Simpson, J., Smith, J., 1998. Ionic polymer-metal composites (IPMCs) as biomimetic sensors, actuators and artificial muscles-a review. Smart Mater. Struct. 7, R15.

Sharafi, S., Li, G., 2015. A multiscale approach for modeling actuation response of polymeric artificial muscles. Soft matter 11, 3833-3843.

Shigley, J.E., Mischke, C.R., Budynas, R.G., Liu, X., Gao, Z., 1989. Mechanical engineering design. McGraw-Hill, New York.

Stroganov, V., Al-Hussein, M., Sommer, J.-U., Janke, A., Zakharchenko, S., Ionov, L., 2015. Reversible thermosensitive biodegradable polymeric actuators based on confined crystallization. Nano Lett. 15, 1786-1790.

Suhai, S., 1983. Ab initio calculation of polyethylene deformation including electron correlation effects. J. Polym. Sci., Part B: Polym. Phys. 21, 1341-1346.

Sze, G.M., Spruiell, J.E., White, J.L., 1976. The influence of drawing, twisting, heat setting, and untwisting on the structure and mechanical properties of melt - spun high - density polyethylene fiber. J. Appl. Polym. Sci. 20, 1823-1847.

Tashiro, K., Kobayashi, M., Tadokoro, H., 1978. Calculation of three-dimensional elastic constants of polymer crystals. 2. Application to orthorhombic polyethylene and poly (vinyl alcohol). Macromolecules 11, 914-918.

Van der Heijden, G., Thompson, J., 2000. Helical and localised buckling in twisted rods: a unified analysis of the symmetric case. Nonlinear Dyn. 21, 71-99.

Wayman, C., 1980. Some applications of shape-memory alloys. JOM 32, 129-137. 
Xiong, B., Lame, O., Chenal, J.-M., Rochas, C., Seguela, R., Vigier, G., 2015. Amorphous Phase Modulus and Micro-Macro Scale Relationship in Polyethylene via in Situ SAXS and WAXS. Macromolecules 48, 2149-2160.

Yang, Q., Li, G., 2015. Temperature and rate dependent thermomechanical modeling of shape memory polymers with physics based phase evolution law. Int. J. Plast. DOI:10.1016/j.ijplas.2015.09.005.

Yip, M.C., Niemeyer, G., 2015. High-performance robotic muscles from conductive nylon sewing thread, Robotics and Automation (ICRA), 2015 IEEE International Conference on. IEEE, pp. 2313-2318.

Zhang, P., Li, G., 2015. Healing-on-demand composites based on polymer artificial muscle. Polym. 64, 29-38.

Zhang, P., Li, G., 2916. Fishing line artificial muscle reinforced composite for impact mitigation and on-demand damage healing. J. Compos. Mater., (in press, 2016) DOI: 10.1177/0021998316636454.

Zhang, Q., Bharti, V., Zhao, X., 1998. Giant electrostriction and relaxor ferroelectric behavior in electron-irradiated poly (vinylidene fluoride-trifluoroethylene) copolymer. Science 280, 2101-2104.

Zhao, Z.-L., Zhao, H.-P., Wang, J.-S., Zhang, Z., Feng, X.-Q., 2014. Mechanical properties of carbon nanotube ropes with hierarchical helical structures. J. Mech. Phys. Solids 71, 6483. 